\title{
Automated, Adaptive Guidance for K-12 Education
}

\begin{abstract}
This paper distinguishes features of automated adaptive guidance used in K-12 instructional settings and recommends directions for design. We use meta-analysis to synthesize 24 independent comparisons between automated adaptive guidance and guidance provided during typical teacher-led instruction, and 29 comparisons that isolate effects of specific adaptive guidance design features in computer-based instruction. We find automated adaptive guidance to be significantly more effective than guidance provided in typical instruction, particularly for students with low prior knowledge. Automated adaptive guidance is most effective when students are generating and integrating ideas (e.g. writing essays, making concept diagrams) as opposed to selecting from given options. Guidance that promoted self-monitoring was more likely to improve learning outcomes than guidance that addressed only content knowledge. Our findings have implications for researchers who investigate K-12 teaching and learning, designers who create and refine instructional materials using automated guidance, and practitioners who deliver or customize instruction featuring automated guidance.
\end{abstract}

Keywords: technology, assessment, automated scoring, automated guidance, pre-college education

\section{Introduction}

Engaging students in reflecting on their ideas, using evidence to sort out their ideas, and constructing arguments and explanations to integrate ideas, is known to foster deep understanding (Bransford, Brown, \& Cocking, 1999; Furtak, Seidel, Iverson, \& Briggs, 2012; Minner, Levy, \& Century 2010). However typical K-12 instruction relies on a combination of lectures and worksheets designed to transmit superficial knowledge rather than support the construction of deep understanding. Furthermore, students are often summatively assessed with multiple-choice tests that encourage the recall of facts without conceptual understanding (Liu, Lee \& Linn, 2011; Shepard, 2008). The classroom emphasis on memorization rather than inquiry and summative rather than formative assessment is due in large part to the challenges associated with guiding diverse students. Posing questions that elicit students' ideas, and providing individualized guidance to address the variety of those ideas, require more time and expertise than many K-12 teachers have. Pre-college instructors often have 5 or 6 classes with up to 40 students each. They often hold multiple-subject credentials and teach outside of their specialty, especially in the fields of science, technology, engineering and mathematics (STEM) and in schools that serve low-income populations (NSF, 2008).

The burgeoning of automated scoring technologies and adaptive guidance for students within online learning environments over the past decade offers promise for responding to this challenge. These tools can help teachers guide students by scoring student work on assessments embedded in online curricula and automating individualized guidance. Such tools may free teachers from the task of evaluating all of their student responses, and allow them to focus more of their efforts on helping students who require further, more complex assistance. 
As automated scoring and guidance technologies continue to evolve, so does their potential to encourage students to deepen their explanations - and consequently, their understanding - of complex ideas. Whereas automated guidance was once used primarily for problems with a single right answer, advanced tools can now diagnose student responses on an array of open-ended, generative activities. Researchers are using natural language processing techniques to identify ideas from students' essays, advanced algorithms to analyze conceptual flaws in student-generated representations, and learning sciences principles to diagnose self-monitoring practices in students' logged interactions with the computer. Researchers are investigating designs for effective guidance that can be automatically delivered to students based on these scored responses and practices.

Over the past three decades automated guidance tools have been used and researched primarily in post-secondary contexts, particularly conceptual physics and computer science (e.g. Smith \& Sherwood, 1976; Van Lehn, 2011). A review of intelligent tutors used in college settings found that student dialogue with a computer tutor was nearly as effective as a dialogue with a human tutor in helping students solve problems in STEM. Both resulted in an average effect size of .7 across comparison studies (VanLehn, 2011).

Automated scoring and guidance tools are now entering the K-12 realm. Researchers are developing and investigating automated guidance tools across K-12 domains such as Language Arts (e.g. Summary Street, Wade-Stein \& Kintsch, 2004), Algebra and Geometry (e.g. Cognitive Tutors, Koedinger \& Aleven, 2007) and Science (e.g. Betty's Brain, Leelawong \& Biswas, 2008). However, reviews of automated guidance in the last 15 years have focused on intelligent tutors in post-secondary contexts (VanLehn, 2011) or Cognitive Tutors exclusively (Koedinger \& Aleven, 2007). With the increasing size and diversity of K-12 classrooms, the availability of robust virtual learning environments, greater access in schools to computers, and stable internet connections, there is now both a need and an opportunity to examine automated guidance technologies in pre-college settings.

The K-12 setting poses challenges that are distinct from those in adult education. First, K12 schooling is mandatory, while adult education is elective. As a result, K-12 students are more diverse regarding their motivation to learn, prior content knowledge, and repertoire of learning strategies. Moreover, K-12 students' success is more reliant on the relationship between student and teacher than the success of typical, higher education students. The K-12 environment offers many instructional supports that might interact with automated scoring. This stands in contrast to the few additional supports in the controlled laboratory environments typically used for experimental research on adult learning. Understanding specifically how K-12 instructional supports can interact with the automated guidance is essential to designing successful approaches to providing automated guidance and will have broad ranging implications for K-12 education.

In this paper, we use meta-analysis to synthesize current research on automated, adaptive guidance implemented across domains in K-12 classrooms. Two research questions guide our analysis:

- Does automated guidance add value to typical instruction for K-12 student learning? 
- What specific features of automated guidance enhance K-12 student learning?

We describe our approach and the findings from our meta-analysis, and consider implications for classroom instruction. We end by relating our findings to the previous research on guidance and learning. Finally, we discuss critical issues for the field of automated scoring and guidance technologies.

\subsection{Conceptual frameworks for guidance design}

Guidance studies fall into three main categories: (1) Characterization of teachers' guidance practices based on observations of teachers in K-12 classrooms, (2) designbased research exploring the value of different features of automated guidance technologies, and (3) experimental studies measuring the comparative benefits of different kinds of guidance on learning outcomes. Each of these types of studies examines the effectiveness of guidance designed from varied conceptual frameworks.

\subsubsection{Teachers' classroom guidance practices}

In their landmark review of more than $250 \mathrm{~K}-12$ classroom studies, Black and Wiliam (1998) concluded that one of the most powerful ways to increase learning gains is for teachers to provide frequent, individualized face-to-face guidance for students. They found particular benefits for low achieving students. Much of this research involved close observation by researchers of teachers' classroom practices over extended periods of time. More recent research on teachers' guidance practices has used a similar methodology. Leinhardt and Steele (2005) for example followed a middle school teacher for 10 lessons and documented the pedagogical strategies she used to help students build mathematical knowledge. Likewise, Herrenkohl, Tasker and White (2011) followed two elementary school teachers for 15 class periods and videotaped their conversations with students as they investigated solar ovens. These and other researchers (e.g., Black \& Wiliam, 1995, Gerard, Spitulnik, \& Linn, 2010; Ruiz-Primo \& Furtak, 2005; van Zee \& Minstrell, 1997) observe how teachers used classroom assessment conversations over multiple lessons to improve learning outcomes. The teachers elicited students' reasoning by assigning generative tasks, then followed up with metacognitive prompts to help students evaluate the validity and strength of their own understanding. Effective teachers were also flexible with their guidance. Rather than executing a predetermined instructional routine, effective teachers were able to customize and vary the formats of their guidance depending on students' individual needs, even while maintaining progress of the class as a whole.

In these studies, guidance that promoted reflection and self-monitoring by encouraging students to make connections between their prior knowledge and the new ideas presented in instruction resulted in significantly greater learning gains than either procedural guidance or pointing out incorrect ideas. These findings are consistent with the tenets of constructivist learning perspectives (DiSessa, 2006; Linn \& Eylon, 2011). Effective guidance is individualized, promotes reflection, and encourages students to distinguish among ideas. In our meta-analysis, one aspect of automated guidance we examine is whether promoting metacognition is more effective than merely correcting erroneous ideas. 


\subsubsection{Post-secondary research on intelligent tutors}

Post-secondary research on intelligent tutors illustrates the value of immediate feedback, but raises questions. Kluger and DeNisi (1996) reviewed 607 studies that compared student performance when given instruction without feedback, with instruction that included feedback delivered either by a computer or by a teacher. They found that while feedback interventions on average had positive impacts on student performance $(d=.41)$, more than a third of those interventions had detrimental impacts. Many studies show that computerized feedback delivered immediately to students improves outcomes (Anderson, Corbett, Koedinger, \& Pelletier, 1995), while others suggest that immediate feedback hinders students' monitoring skills (Moreno \& Valdez, 2005), encourages mindless clicking rather than thoughtful reconsideration of information (Baker et al., 2008), and gives students an overly optimistic view of their understanding (Bjork, 1994).

The research on intelligent tutors demonstrates the value of research designs comparing alternative forms of guidance. Studies identify effective features. Several of these studies suggest that effective guidance encourages students to identify criteria for distinguishing among ideas rather than simply directing students to accept the most valid idea (Arnott, Hastints, \& Albitron, 2008; Azevedo, 2004; Graesser, McNamara, \& Van Lehn, 2005). These studies additionally find that scaffolding students' problem-solving process, such as through step-based and substep-based tutoring systems, is more effective than diagnosing the accuracy of their answers, such as through answer-based tutoring systems (VanLehn, 2011). These findings echo the features of effective teacher guidance. Guiding students to sort out their initial ideas and those presented by instruction and then refine their view appears to be more successful than directing students to the right answer.

\subsubsection{The added value of automated guidance}

Numerous challenges exist for K-12 teachers to provide high-quality, individualized guidance to students during the course of instruction (Leinhardt \& Steele, 2005).

Teachers report a lack of time to generate detailed guidance on all of their student's work. In some cases, teachers also report a lack of experience with the range of student ideas on the topic to be able to anticipate and quickly formulate effective, individualized guidance. Ruiz-Primo \& Furtak (2007) found that teacher guidance for students' scientific explanations focused more on procedural guidance than knowledge generation. This echoes Black \& Wiliam's (1998) finding that teacher guidance is more often geared toward improving performance on tests than toward addressing students' individual learning needs. Moreover, those teachers most effective in their guidance have had to develop additional strategies that would enable them to deliver that guidance, such as frequently circling and monitoring the classroom (Gerard et al., 2010). These logistic challenges limit teachers' ability to attend to all students who require assistance. Based on these reports of classroom challenges and results of empirical studies on automated guidance, researchers suggest that automated scoring technologies might, by diagnosing and guiding students' reasoning, complement teachers and help them become more successful in their practice. 
Through relatively simple interventions, researchers have begun to develop ways by which computer-based guidance might serve the individual needs of K-12 students in a way that would otherwise be impractical by the teacher alone. In this paper, we synthesize studies on the learning impacts of automated scoring and guidance in K-12 classrooms. Our meta-analysis of these studies addresses two research goals: First, to understand the value added of automated guidance for teachers in K-12 settings; and second, to identify the most promising features of automated guidance for future development and research.

\section{Methods}

\subsection{Identification of studies}

We began by searching the education research databases ERIC, PsycINFO and Google Scholar. We refined our search terms to capture the features of interest related to automated guidance, including the technology (computer, web-based, adaptive), its function for providing guidance (feedback, formative assessment, tutor), and the setting of its use (education). We also searched recent issues of peer-reviewed journals that publish K-12 instructional comparison studies of technology-based interventions and use rigorous research methods. These included: Journal of Research in Science Teaching, Science, International Journal of Artificial Intelligence in Education, Journal of the Learning Sciences, and Journal of Educational Psychology. Additionally, we searched proceedings of the International Conference of the Learning Sciences, Computer Supported Collaborative Learning conference, International Conference on Intelligent Tutoring Systems, and Artificial Intelligence in Education series. Finally, we requested unpublished papers from research groups known to investigate automated guidance, and searched the reference list of each paper for additional candidates. To account for the impact of modern technologies during the past decade, we limited our search to papers published from 2000 to 2014 .

Once we had gathered a set of papers from this initial search, we read their abstracts to filter out those that did not meet our criteria for inclusion. We selected studies that (1) offered empirical data, (2) were conducted with students in a K-12 educational setting, (3) investigated computer-based automated, adaptive guidance, and (4) used the same content and instructional design in the comparison condition. We used a broad definition of automated, adaptive guidance to capture all forms of computer-based individualized support for K-12 students. We considered automated, adaptive guidance to include guidance that occurred within a computer-based instructional sequence. Such guidance involved two components: the computer-scoring of students' work, and the computerdelivery of a response to students' work based on the automated scores. Examples include text-based hints, encouragement to reconsider and revise written work, and new activities tailored to give students additional practice on particular tasks.

We examined the methodological details of the selected studies to determine whether they offered the necessary information to be included in a meta-analysis (e.g., N, means, 
$\mathrm{SD}, \mathrm{t}$ - and F-values). When relevant data appeared to have been collected but were not reported in the paper, or when we required clarification of any methodological details, we contacted the paper authors and asked for additional information. Ultimately, our search and selection process yielded 41 studies and 57 independent effect sizes.

\subsection{Coding of Study Characteristics}

We next read the papers in their entirety to identify emergent variables that might potentially influence the effect of the automated guidance on learning outcomes. Many of these variables, although theoretically important, had to eventually be collapsed or otherwise eliminated because they showed too little variation in learning outcomes across the studies. For example, the variable "provided the student with the correct answer" is a potentially meaningful feature of automated guidance. Its effectiveness relative to guidance that encourages reflection without giving the correct answer, for example, is an empirical question with real consequences for instructional design. However, this variable was only investigated by 2 of the 41 studies, and moreover, the learning outcomes that were a result of this variable showed little variation across those studies. We thus had to collapse this variable into a larger category of guidance ("kinds of automated guidance") in order to include it in the meta-analysis. Variables investigated but lacking sufficient meta-analytic data across studies included modality (e.g., text vs. image vs. voice), timing (e.g., immediate or delayed), automated scoring technique (e.g., natural language processing techniques or key words), student collaboration in use of guidance (e.g., individual vs. with a partner), and the attributed source of the guidance (e.g., avatar vs. no avatar).

The final list of moderator variables with sufficient meta-analytic data for analysis fell into three categories (Table 1): Student characteristics, which included: (1) grade level, (2) domain, and (3) learners' level of prior knowledge; study design characteristics, which included (1) the total duration of the intervention, (2) the nature of comparison conditions; (3) the type of activity on which students received automated guidance, (3) the form of the outcome assessment, (4) the nature of what was assessed by the automated system, (5) the timing of the outcome assessment; and characteristics of the automated guidance, which included (1) the technology used in automated scoring, (2) the kind of automated guidance given to students (2) whether the guidance encouraged self-monitoring, and (3) whether the guidance made use of adaptive problems.

Within each of these broad categories, we further divided the studies into a number of sub-categories. We iterated on the organization of these subcategories several times, defining and collapsing them as necessary, in order to capture a sufficient number of studies $(\geq 4)$ in each for the purposes of the meta-analysis (Borenstein, Hedges, Higgins, \& Rothstein, 2009). 
Automated, Adaptive Guidance for K-12 Education

\section{Table 1. Moderator Variables and Coding Categories}

\begin{tabular}{|c|c|}
\hline Moderator Variables & Sub Categories \\
\hline & Student Characteristics \\
\hline Grade & $\begin{array}{l}\text { 1. Elementary and Middle school. Students are in grades 6-8 or equivalent. } \\
\text { 2. High school. Students are in grades 9-12 or equivalent. }\end{array}$ \\
\hline Domain & $\begin{array}{l}\text { 1. Mathematics. Includes algebra, calculus, geometry. } \\
\text { 2. Literacy and Social Studies. Includes English Language Arts, geography, history } \\
\text { 3. } \\
\end{array}$ \\
\hline \multirow[t]{2}{*}{ Prior Knowledge } & $\begin{array}{l}\text { 1. Low. Students scored low on pretest. } \\
\text { 2. Mixed or medium. Students tested vary in their pretest performance. } \\
\text { 3. High. Students scored high on pretests. }\end{array}$ \\
\hline & Study design characteristics \\
\hline Duration of the Intervention & $\begin{array}{l}\text { 1. One class period or less. The intervention took place within the time frame of a 50- } \\
\text { minute class period. } \\
\text { 2. More than one class period. The intervention was extended over a period of time greater } \\
\text { than a 50-minute class period, or over multiple sessions. }\end{array}$ \\
\hline Type of control & $\begin{array}{l}\text { 1. Teacher led instruction. The intervention is compared to regular teacher-led instruction. } \\
\text { 2. Worksheets no guidance. Students independently complete worksheets on the subject, } \\
\text { and receive no guidance. } \\
\text { 3. Computer program with fixed guidance or teacher-generated guidance. Often } \\
\text { contrasted with computer adaptive guidance. }\end{array}$ \\
\hline $\begin{array}{l}\text { Learning Activity on which Student } \\
\text { Gets Guidance }\end{array}$ & $\begin{array}{l}\text { 1. Written explanation. Students type an explanation of their thinking in response to a } \\
\text { specific prompt. } \\
\text { 2. Drawing or Concept diagram. Students draw a visual representation of ideas. Task is } \\
\text { constrained by use of pre-given stamps or icons. } \\
\text { 3. Mathematical operations or formulas. Students use formulas to solve given problems in } \\
\text { mathematics, chemistry, or physics. } \\
\text { 4. Multiple-choice. Students select one or more options from a set of given options. }\end{array}$ \\
\hline $\begin{array}{l}\text { What is assessed in Outcome } \\
\text { Measure }\end{array}$ & $\begin{array}{l}\text { 1. Content. Assesses that students' choice or response provides correct, valid information. } \\
\text { 2. Strategies. Assesses the students' approaches to solving a problem. } \\
\text { 3. Writing Structure. Assesses that students' essays include a proper introduction, body, } \\
\text { and conclusion; or an argument contain appropriate evidence to support a claim. }\end{array}$ \\
\hline Form of Outcome Measure & $\begin{array}{l}\text { 1. Selection. Multiple choice questions. } \\
\text { 2. Generation. Student-generated representations. } \\
\text { 3. Mixed. Combination of selection and generation assessments. }\end{array}$ \\
\hline \multirow[t]{2}{*}{ Timing of Outcome Measure } & $\begin{array}{l}\text { 1. Embedded. An assessment found within the intervention. } \\
\text { 2. PrePost. A pre and posttest administered immediately before and after the intervention. } \\
\text { 3. Delayed Post. An assessment administered two or more weeks after the intervention. } \\
\text { 4. Mixed. Combination of embedded, prepost and, or delayed post. }\end{array}$ \\
\hline & Automated guidance characteristics \\
\hline Technology Used for Auto-Scoring & $\begin{array}{l}\text { 1. Natural Language Processing. Use of machine learning and data mining tools to score } \\
\text { level of conceptual accuracy and, or writing coherence in student generated text. } \\
\text { 2. Correct/Incorrect algorithms. Compares student answer to pre-determined correct } \\
\text { answer and judges right or wrong. }\end{array}$ \\
\hline Kinds of automated guidance & $\begin{array}{l}\text { 1. Specific guidance. Informs student if answer is correct or incorrect and provides student } \\
\text { with correct answer. May also identify errors. } \\
\text { 2. Conceptual guidance. Prompts students to consider their ideas relative to identified } \\
\text { missing or non-normative concept. Does not provide correct answer. } \\
\text { 3. Self-Monitoring guidance. Encourages students to reflect on their understanding and the } \\
\text { process used to achieve their response. } \\
\text { 4. Multiple forms of guidance can include any two or more of the kinds above. }\end{array}$ \\
\hline Adaptive problems & $\begin{array}{l}\text { 1. Yes. Based on their automatically scored responses, students are assigned to complete } \\
\text { new problems. } \\
\text { 2. No. Students either receive a new, predetermined activity that is not assigned based on } \\
\text { their prior work; or are asked to revise their prior work. }\end{array}$ \\
\hline Encourages self-monitoring & $\begin{array}{l}\text { 1. Yes. Program includes guidance to encourages students to reflect on their understanding } \\
\text { and the process used to achieve their response } \\
\text { 2. No. Program does not include guidance to prompt self-monitoring. }\end{array}$ \\
\hline
\end{tabular}


After coding the studies, we divided them into two categories based on their research designs:

1. Automated adaptive guidance vs. typical instruction [Table 2]. This category compared automated, adaptive guidance with what authors of the studies labeled typical instruction, or classroom instruction that lacked automated guidance. Typical instruction included any manner of classroom learning activity that occurred in the absence of automated adaptive guidance. Examples include students' independent completion of worksheets, teacher-facilitated instruction and guidance, and computer-based instruction with no automated guidance.

Table 2. Automated Guidance v. Typical Instruction Studies, Comparisons and Effect Size

\begin{tabular}{|c|c|c|c|}
\hline Citation & $\begin{array}{l}\text { Comparison Description } \\
\text { (Treatment v. Control) }\end{array}$ & $\begin{array}{l}\text { Cohen's } \\
\text { criterion }\end{array}$ & Hedges g \\
\hline $\begin{array}{l}\text { Chin, D. B., Dohmen, I. M., Cheng, B. H., Oppezzo, M. A., Chase, C. C., } \\
\text { \& Schwartz, D. L. (2010). Preparing students for future learning with } \\
\text { Teachable Agents. [Feature]. Educational Technology Research and } \\
\text { Development, 58(6), 649-669. }\end{array}$ & $\begin{array}{l}\text { Computer Tutor vs. } \\
\text { Computer with No Guidance }\end{array}$ & $\mathrm{L}$ & 0.96 \\
\hline $\begin{array}{l}\text { Beal, C. R., Walles, R., Arroyo, I., \& Woolf, B. P. (2007). On-line } \\
\text { tutoring for Math Achievement Testing: A Controlled Evaluation. } \\
\text { [Feature]. Journal of Interactive Online Learning, 6(1), 43-55. }\end{array}$ & $\begin{array}{l}\text { Computer Tutor vs. Teacher- } \\
\text { led Instruction (Low Prior)* }\end{array}$ & $\mathrm{L}$ & 0.85 \\
\hline $\begin{array}{l}\text { Poulsen, R., Hastings, P., \& Allbritton, D. (2007). Tutoring bilingual } \\
\text { students with an automated reading tutor that listens. [Feature]. } \\
\text { Journal of Educational Computing Research, 36(2), 191-221. }\end{array}$ & $\begin{array}{l}\text { Reading Tutor vs. Teacher-led } \\
\text { Instruction }\end{array}$ & M & 0.76 \\
\hline $\begin{array}{l}\text { Mendicino, M., Razzaq, L., \& Heffernan, N. T. (2009). A comparison of } \\
\text { traditional homework to computer-supported homework. [Feature]. } \\
\text { Journal of Research on Technology in Education, 41(3), 331-359. }\end{array}$ & $\begin{array}{l}\text { Computer Tutor vs. Teacher- } \\
\text { led Instruction }\end{array}$ & M & 0.62 \\
\hline $\begin{array}{l}\text { Beal, C. R., Arroyo, I., Cohen, P. R., \& Woolf, B. P. (2010). Evaluation of } \\
\text { AnimalWatch: An intelligent tutoring system for arithmetic and } \\
\text { fractions. Journal of Interactive Online Learning, 9(1), 64-77. }\end{array}$ & $\begin{array}{l}\text { Adaptive Computer } \\
\text { Instruction vs. Teacher-led } \\
\text { Instruction (Low Prior) } \\
\end{array}$ & M & 0.6 \\
\hline $\begin{array}{l}\text { Hung, P.-H., Hwang, G.-J., Lin, Y.-F., Wu, T.-H., \& Su, I.-H. (2013). } \\
\text { Seamless connection between learning and assessment applying } \\
\text { progressive learning tasks in mobile Ecology inquiry. Educational } \\
\text { Technology \& Society, } 16 \text { (1), 194-205. }\end{array}$ & $\begin{array}{l}\text { Adaptive Guidance + } \\
\text { Feedback in Computer } \\
\text { Program vs. Teacher-led } \\
\text { Instruction }\end{array}$ & M & 0.59 \\
\hline $\begin{array}{l}\text { Huang, T.-H., Liu, Y.-C., \& Shiu, C.-Y. (2008). Construction of an online } \\
\text { learning system for decimal numbers through the use of cognitive } \\
\text { conflict strategy. [Feature]. Computers \& Education, 50(1), 61-76. }\end{array}$ & $\begin{array}{l}\text { Computer Tutor vs. Typical } \\
\text { Worksheets with No } \\
\text { Feedback (Low Prior) }\end{array}$ & M & 0.52 \\
\hline $\begin{array}{l}\text { Kalyuga, S., \& Sweller, J. (2005). Rapid dynamic assessment of } \\
\text { expertise to improve the efficiency of adaptive e-learning. [Feature]. } \\
\text { Educational Technology Research and Development, 53(3), 83-93. }\end{array}$ & $\begin{array}{l}\text { Adaptive Problems v. Same } \\
\text { Computer Program with } \\
\text { Fixed Problems }\end{array}$ & M & 0.52 \\
\hline $\begin{array}{l}\text { Schoppek, W., \& Tulis, M. (2010). Enhancing arithmetic and word- } \\
\text { problem solving skills efficiently by individualized computer-assisted } \\
\text { practice. [Feature]. The Journal of Educational Research, 103(4), 239- } \\
252 .\end{array}$ & $\begin{array}{l}\text { Computer Tutor vs. Teacher- } \\
\text { led Instruction }\end{array}$ & M & 0.52 \\
\hline $\begin{array}{l}\text { Koedinger, K. R., McLaughlin, E. A., \& Heffernan, N. T. (2010). A quasi- } \\
\text { experimental evaluation of an on-line formative assessment and } \\
\text { tutoring system. Journal of Educational Computing Research, 43(4), } \\
\text { 489-510. }\end{array}$ & $\begin{array}{l}\text { Computer Tutor vs. Typical } \\
\text { Textbook Instruction (Special } \\
\text { Ed) }\end{array}$ & M & 0.49 \\
\hline $\begin{array}{l}\text { Molenaar, I., Roda, C., Boxtel, C., Sleegers, P. (2012). Dynamic } \\
\text { scaffolding of socially regulated learning in a computer based learning } \\
\text { environment. Computers and Education, } 59,515-523 \text {. }\end{array}$ & $\begin{array}{l}\text { Cognitive \& Metacognitive } \\
\text { Feedback v. Computer } \\
\text { Program with No Feedback } \\
\end{array}$ & M & 0.49 \\
\hline $\begin{array}{l}\text { Chin, D. B., Dohmen, I. M., Cheng, B. H., Oppezzo, M. A., Chase, C. C., } \\
\text { \& Schwartz, D. L. (2010). Preparing students for future learning with } \\
\text { Teachable Agents. [Feature]. Educational Technology Research and } \\
\text { Development, 58(6), 649-669. }\end{array}$ & $\begin{array}{l}\text { Computer Tutor vs. } \\
\text { Computer with No Feedback }\end{array}$ & M & 0.44 \\
\hline $\begin{array}{l}\text { Huang, T.-H., Liu, Y.-C., \& Shiu, C.-Y. (2008). Construction of an online } \\
\text { learning system for decimal numbers through the use of cognitive } \\
\text { conflict strategy. [Feature]. Computers \& Education, 50(1), 61-76. }\end{array}$ & $\begin{array}{l}\text { Computer Tutor vs. Teacher- } \\
\text { led Instruction (High Prior) }\end{array}$ & M & 0.41 \\
\hline Huang, T.-H., Liu, Y.-C., \& Shiu, C.-Y. (2008). Construction of an online & Computer Tutor vs. Teacher- & $\mathrm{M}$ & 0.41 \\
\hline
\end{tabular}




\begin{tabular}{|c|c|c|c|}
\hline $\begin{array}{l}\text { learning system for decimal numbers through the use of cognitive } \\
\text { conflict strategy. [Feature]. Computers \& Education, 50(1), 61-76. }\end{array}$ & led Instruction (Med Prior) & & \\
\hline $\begin{array}{l}\text { McElhaney, K., Gerard, L., Rafferty, A., \& Linn, M.C., (2012). } \\
\text { Comparing the benefits of automated and teacher feedback on } \\
\text { sudent-generated molecular representations, Paper presented at } \\
\text { American Education Research Association, San Francisco, CA. }\end{array}$ & $\begin{array}{l}\text { Computer Assigned } \\
\text { Knowledge Integration } \\
\text { Feedback v. Same Computer } \\
\text { Program with Teacher } \\
\text { Generated Feedback (Low } \\
\text { Prior) }\end{array}$ & M & 0.38 \\
\hline $\begin{array}{l}\text { White, L., Hixson, N., D'Brot, J., Perdue, J., Foster, S. \& Rhudy, V. } 2010 . \\
\text { Research brief: Impact of Writing Roadmap } 2.0 \text { on WESTEST } 2 \text { online } \\
\text { writing assessment scores. Charleston, West Virginia: West Virginia } \\
\text { Department of Education. }\end{array}$ & $\begin{array}{l}\text { Writing Roadmap v Teacher- } \\
\text { led Instruction }\end{array}$ & M & 0.38 \\
\hline $\begin{array}{l}\text { Tang, J., Rich, C. S., \& Wang, Y. (2012). Technology-enhanced English } \\
\text { language writing assessment in the classroom. Chinese Journal of } \\
\text { Applied Linguistics, 35(4), 385-399. }\end{array}$ & $\begin{array}{l}\text { Writing Roadmap v. Teacher- } \\
\text { led Instruction }\end{array}$ & $s$ & 0.31 \\
\hline $\begin{array}{l}\text { Rich, C. S. (2012). The Impact of Online Automated Writing } \\
\text { Evaluation: A Case Study from Dalian. Chinese Journal of Applied } \\
\text { Linguistics, 35(1), 63-79. }\end{array}$ & $\begin{array}{l}\text { Writing Roadmap v. Teacher- } \\
\text { led Instruction }\end{array}$ & S & 0.3 \\
\hline $\begin{array}{l}\text { Koedinger, K. R., McLaughlin, E. A., \& Heffernan, N. T. (2010). A quasi- } \\
\text { experimental evaluation of an on-line formative assessment and } \\
\text { tutoring system. Journal of Educational Computing Research, 43(4), } \\
489-510\end{array}$ & $\begin{array}{l}\text { Computer Tutor vs. Typical } \\
\text { Textbook Instruction (High } \\
\text { Prior) }\end{array}$ & $\mathrm{N}$ & 0.08 \\
\hline $\begin{array}{l}\text { Rafferty, A. N., Gerard, L., McElhaney, K., \& Linn, M. C. (2014). } \\
\text { Promoting student learning through automated formative guidance } \\
\text { on chemistry drawings. Proceedings of the International Conference of } \\
\text { the Learning Sciences (ICLS) } 2014 \text { (pp. 386-393). }\end{array}$ & $\begin{array}{l}\text { Computer Assigned } \\
\text { Knowledge Integration } \\
\text { Feedback v. Same Computer } \\
\text { Program with Teacher } \\
\text { Generated Feedback }\end{array}$ & $\mathrm{N}$ & 0.04 \\
\hline $\begin{array}{l}\text { Gerard, L., Linn, M.C. \& Liu, O.L. (2012). How well can the computer } \\
\text { assign feedback on student generated explanations? A comparison } \\
\text { study of computer and teacher adaptive guidance. Paper presented at } \\
\text { American Education Research Association, San Francisco, CA. }\end{array}$ & $\begin{array}{l}\text { Computer Assigned v. } \\
\text { Teacher Assigned Knowledge } \\
\text { Integration Feedback }\end{array}$ & $\mathrm{N}$ & -0.04 \\
\hline $\begin{array}{l}\text { Beal, C. R., Arroyo, I., Cohen, P. R., \& Woolf, B. P. (2010). Evaluation of } \\
\text { AnimalWatch: An intelligent tutoring system for arithmetic and } \\
\text { fractions. Journal of Interactive Online Learning, } 9(1), 64-77\end{array}$ & $\begin{array}{l}\text { Adaptive Computer } \\
\text { Instruction vs. Teacher-led } \\
\text { Instruction (High Prior) } \\
\end{array}$ & $\begin{array}{l}\text { S [in favor } \\
\text { of } \\
\text { control] }\end{array}$ & -0.21 \\
\hline $\begin{array}{l}\text { Beal, C. R., Walles, R., Arroyo, I., \& Woolf, B. P. (2007). On-line } \\
\text { tutoring for Math achievement testing: A controlled evaluation. } \\
\text { [Feature]. Journal of Interactive Online Learning, 6(1), 43-55. }\end{array}$ & $\begin{array}{l}\text { Computer Tutor vs. Teacher- } \\
\text { led Instruction (High Prior) }\end{array}$ & $\begin{array}{l}\text { S [in favor } \\
\text { of } \\
\text { control] }\end{array}$ & -0.28 \\
\hline $\begin{array}{l}\text { McElhaney, K., Gerard, L., Rafferty, A., \& Linn, M.C., (2012). } \\
\text { Comparing the benefits of automated and teacher feedback on } \\
\text { student-generated molecular Representations. Paper presented at } \\
\text { American Education Research Association, San Francisco, CA. }\end{array}$ & $\begin{array}{l}\text { Computer Assigned } \\
\text { Knowledge Integration } \\
\text { Feedback v. Same Computer } \\
\text { Program with Teacher } \\
\text { Generated Feedback (High } \\
\text { Prior) }\end{array}$ & $\begin{array}{l}\text { M [in } \\
\text { favor of } \\
\text { control] }\end{array}$ & -0.44 \\
\hline
\end{tabular}

2. Enhanced vs. simple automated adaptive guidance [Table 3]. This category compared different features of automated guidance. To be included in the meta-analysis, the curriculum in which the automated guidance was embedded needed to be the same in both the treatment and control conditions. Enhanced guidance included a particular feature that researchers set out to investigate (a treatment group) that elaborated upon a simpler form of automated guidance (a control group). The variety of comparisons featured in the studies we retrieved made it impossible to devise a consistent definition of enhancement or simplicity. Instead, we defined enhanced guidance relative to the simple guidance that served as its control comparison. This approach permits us to characterize the effectiveness of the enhanced feature being investigated in the studies. For example, Rebolledo-Mendez, Luckin and du Boulay (2011), investigated motivational guidance as an enhancement of the adaptive activities (without motivational guidance) within the learning environment, Ecolab. Meanwhile, Yun, Miller, Baek, Jung, \& Ko (2008) 
compared guidance that encouraged students to make further attempts in addition to only diagnosing correct or incorrect responses. Although one might consider RebolledoMendez et al.'s adaptive activities to be technologically enhanced relative to Yun et al.'s straightforward diagnostic guidance, it is by virtue of their status in their respective experiments that we coded the guidance thus.

Table 3. Automated Enhanced Guidance v. Automated Simpler Guidance Studies, Comparisons and Effect Size

\begin{tabular}{|c|c|c|c|}
\hline Citation & $\begin{array}{l}\text { Comparison Description } \\
\text { (Enhanced v. Simple) }\end{array}$ & $\begin{array}{l}\text { Cohen's } \\
\text { criterion }\end{array}$ & Hedges $\mathrm{g}$ \\
\hline $\begin{array}{l}\text { Leelawong, K., \& Biswas, G. (2008). Designing learning by teaching } \\
\text { agents: The Betty's Brain system. International Journal of Artificial } \\
\text { Intelligence in Education, 18(3), 181-208 }\end{array}$ & $\begin{array}{l}\text { Learn by Teaching Approach } \\
+ \text { Content guidance } v \text {. } \\
\text { Content guidance only }\end{array}$ & $\mathrm{L}$ & 1.43 \\
\hline $\begin{array}{l}\text { Narciss, S. \& Huth, K. (2006). Fostering achievement and motivation } \\
\text { with bug-related tutoring feedback in a computer-based training for } \\
\text { written subtraction. Learning and Instruction,16(4), 310-322. }\end{array}$ & $\begin{array}{l}\text { Auto BRT (bug related } \\
\text { tutoring) v. Auto KCR (right } \\
\text { wrong + correct answer) }\end{array}$ & L & 1.22 \\
\hline $\begin{array}{l}\text { Wade-Stein, D., \& Kintsch, E. (2004). Summary Street: Interactive } \\
\text { computer support for writing. [Feature]. Cognition and Instruction, } \\
22(3), 333-362 \text {. }\end{array}$ & $\begin{array}{l}\text { Detailed feedback on } \\
\text { summary content v. only } \\
\text { feedback on length/spelling }\end{array}$ & L & 0.76 \\
\hline $\begin{array}{l}\text { Aleven, V. \& Koedinger, K. (2002). An effective metacognitive } \\
\text { strategy: learning by doing and explaining with a computer-based } \\
\text { Cognitive Tutor. Cognitive Science, 26, 247-179. }\end{array}$ & $\begin{array}{l}\text { Cog Tutor + Self Explanation } \\
\text { v. Cog Tutor }\end{array}$ & M & .61 \\
\hline $\begin{array}{l}\text { Segedy, Kinnebrew, Biswas (2012). The effect of contextualized } \\
\text { conversational feedback in a complex open-ended learning } \\
\text { environment. Education Technology Research and Development 61, } \\
\text { 71-89. }\end{array}$ & $\begin{array}{l}\text { Conversational } \\
\text { Contextualized Feedback v. } \\
\text { Direct General Feedback }\end{array}$ & $M$ & 0.58 \\
\hline $\begin{array}{l}\text { Rebolledo-Mendez, G., R. , Luckin, R., \& du Boulay, B. (2011). } \\
\text { Designing adaptive motivational scaffolding for a tutoring system In R. } \\
\text { A. Calvo \& S. K. D'Mello (Eds.), New Perspectives on Affect and } \\
\text { Learning Technologies (Vol. 3, pp. 155-168). New York: Springer. }\end{array}$ & $\begin{array}{l}\text { Ecolab Adaptive Activities + } \\
\text { Motivational Feedback v. } \\
\text { Ecolab Adaptive Activities }\end{array}$ & $M$ & 0.54 \\
\hline $\begin{array}{l}\text { Walker, E., Rummel, N., \& Koedinger, K. R. (2011). Using automated } \\
\text { dialog analysis to assess peer tutoring and trigger effective support. In } \\
\text { G. Biswas, S. Bull, J. Kay, A. Mitrovic (Eds.), Proceedings of the 10th } \\
\text { International Conference on Artificial Intelligence in Education (pp. } \\
\text { 385-393). Berlin: Springer. }\end{array}$ & $\begin{array}{l}\text { Adaptive Feedback for Peer } \\
\text { (plus Cog Tutor) v. Fixed Hints } \\
\text { on Demand for Peer (plus } \\
\text { Cog Tutor) }\end{array}$ & $M$ & 0.48 \\
\hline $\begin{array}{l}\text { Ryoo, K. \& Linn, M.C. (2014). Comparison of specific and knowledge } \\
\text { integration automated guidance for concept diagrams in inquiry } \\
\text { instruction. Proceedings of the International Conference of the } \\
\text { Learning Sciences (ICLS) 2014, (pp. 585-587). }\end{array}$ & $\begin{array}{l}\text { Adaptive Knowledge } \\
\text { Integration Feedback v. } \\
\text { Adaptive Specific Feedback }\end{array}$ & $M$ & 0.41 \\
\hline $\begin{array}{l}\text { Yun, S., Miller, P. C., Baek, Y., Jung, J., \& Ko, M. (2008). Improving } \\
\text { recall and transfer skills through vocabulary building in web-based } \\
\text { second language learning: An examination by item and feedback type. } \\
\text { [Feature]. Journal of Educational Technology \& Society, 11(4), 158- } \\
172 .\end{array}$ & $\begin{array}{l}\text { Right Answer + Try Again } \\
\text { Feedback v. Right Answer }\end{array}$ & $M$ & 0.38 \\
\hline $\begin{array}{l}\text { Razzaq, L., Heffernan, N.T. (2006). Scaffolding vs. hints in the } \\
\text { Assistment System. In Ikeda, Ashley \& Chan (Eds.). Proceedings of the } \\
\text { 8th International Conference on Intelligent Tutoring Systems. } \\
\text { Springer-Verlag: Berlin. pp. 635-644. }\end{array}$ & $\begin{array}{l}\text { Adaptive Scaffolding v. Fixed } \\
\text { Hints On Demand }\end{array}$ & $S$ & 0.28 \\
\hline $\begin{array}{l}\text { Shute, V. J., Hansen, E. G., \& Almond, R. G. (2008). You can't fatten a } \\
\text { hog by weighing it - “Or can you? Evaluating an assessment for } \\
\text { learning system called ACED. International Journal of Artificial } \\
\text { Intelligence in Education, 18(4), 289-316. }\end{array}$ & $\begin{array}{l}\text { Elaborated Feedback + } \\
\text { Adaptive Sequencing v. } \\
\text { Simple Feedback + Adaptive } \\
\text { Sequencing }\end{array}$ & $S$ & 0.27 \\
\hline $\begin{array}{l}\text { Dresel, M., \& Haugwitz, M. (2008). A computer-based approach to } \\
\text { fostering motivation and self-regulated learning. [Feature]. The } \\
\text { Journal of Experimental Education, } 77(1), 3-18 \text {. }\end{array}$ & $\begin{array}{l}\text { Attributional Feedback + } \\
\text { Right/Wrong v. Right/Wrong }\end{array}$ & S & 0.22 \\
\hline $\begin{array}{l}\text { Franzke, M., Kintsch, E., Caccamise, D., Johnson, N., \& Dooley, S. } \\
\text { (2005). Summary Street: Computer support for comprehension and } \\
\text { writing. Journal of Educational Computing Research, } 33,53-80\end{array}$ & $\begin{array}{l}\text { Adaptive Feedback v. Word } \\
\text { Processing Feedback }\end{array}$ & S & 0.22 \\
\hline $\begin{array}{l}\text { Kinnebrew, J. S., Biswas, G., \& Sulcer, W. B. (2010). Modeling and } \\
\text { measuring self-regulated learning in teachable agent environments. } \\
\text { Paper presented at the Association for the Advancement of Artificial } \\
\text { Intelligence, Atlanta, GA. }\end{array}$ & $\begin{array}{l}\text { Learn by Teaching Approach } \\
+ \text { Content Guidance v. } \\
\text { Content Guidance only }\end{array}$ & S & 0.2 \\
\hline
\end{tabular}




\begin{tabular}{|c|c|c|c|}
\hline $\begin{array}{l}\text { Timms, M. J. (2007). Using Item Response Theory (IRT) to select hints } \\
\text { in an ITS. Frontiers in Artificial Intelligence and Applications, 158, } 213 .\end{array}$ & $\begin{array}{l}\text { Error Detection + Adaptive } \\
\text { Feedback v. Error Detection }\end{array}$ & $S$ & 0.18 \\
\hline $\begin{array}{l}\text { Razzaq, L. \& Heffernan, N. (2009). To tutor or not to tutor: That is the } \\
\text { question. In Dimitrova, Mizoguchi, du Boulay \& Graesser (Eds.) } \\
\text { Proceedings of the } 2009 \text { Artificial Intelligence in Education } \\
\text { Conference. pp. 457-464. ISO Press, Netherlands. }\end{array}$ & $\begin{array}{l}\text { Step by Step Guidance v. } \\
\text { Worked Our Right Answer } \\
\text { Feedback (Low Pior) }\end{array}$ & $S$ & 0.16 \\
\hline $\begin{array}{l}\text { Meyer, B. J., Wijekumar, K., Middlemiss, W., Higley, K., Lei, P. W., } \\
\text { Meier, C., \& Spielvogel, J. (2010). Web-based tutoring of the structure } \\
\text { strategy with or without elaborated feedback or choice for fifth and } \\
\text { seventh grade readers. Reading Research Quarterly, 45(1), 62-92. }\end{array}$ & $\begin{array}{l}\text { Elaborated v. Simple } \\
\text { Computer Feedback (Low } \\
\text { Prior) }\end{array}$ & $S$ & 0.15 \\
\hline $\begin{array}{l}\text { Meyer, B. J. F., Wijekumar, K. K., \& Lin, Y.-C. (2011). Individualizing a } \\
\text { Web-Based Structure Strategy Intervention for Fifth Graders' } \\
\text { Comprehension of Nonfiction. [Feature]. Journal of Educational } \\
\text { Psychology, 103(1), 140-168. }\end{array}$ & $\begin{array}{l}\text { Simple Feedback + Adaptive } \\
\text { Problems v. Simple Feedback } \\
\text { (Low prior) }\end{array}$ & $S$ & 0.15 \\
\hline $\begin{array}{l}\text { McLaren, B.; DeLeeuw, K.,Mayer, R. (2011). Polite web-based } \\
\text { intelligent tutors: Can they improve learning in classrooms? } \\
\text { Computers \& Education, 56(3), 574-584. }\end{array}$ & $\begin{array}{l}\text { Polite Feedback v. Direct } \\
\text { Feedback (Low Prior) }\end{array}$ & $S$ & 0.13 \\
\hline $\begin{array}{l}\text { Heffernan, N., Koedinger, K., \& Razzaq, L. (2008). Expanding the } \\
\text { model tracing architecture: a 3rd generation intelligent tutor for } \\
\text { algebra symbolization, International Journal of Al in Education, 18, } \\
\text { 153-178. }\end{array}$ & $\begin{array}{l}\text { Tutor Explain in English } \\
\text { Strategy v. Showing Correct } \\
\text { Answer }\end{array}$ & $S$ & 0.12 \\
\hline $\begin{array}{l}\text { McLaren, B., DeLeeuw, K., Mayer, R. (2011). Polite web-based } \\
\text { intelligent tutors: Can they improve learning in classrooms? } \\
\text { Computers \& Education, 56(3), 574-584. }\end{array}$ & $\begin{array}{l}\text { Polite Feedback v. Direct } \\
\text { Feedback (High Prior) }\end{array}$ & $S$ & 0.11 \\
\hline $\begin{array}{l}\text { Meyer, B. J. F., Wijekumar, K. K., \& Lin, Y.-C. (2011). Individualizing a } \\
\text { web-based structure strategy intervention for fifth graders' } \\
\text { comprehension of nonfiction. [Feature]. Journal of Educational } \\
\text { Psychology, 103(1), 140-168. }\end{array}$ & $\begin{array}{l}\text { Simple Feedback + Adaptive } \\
\text { Problems v. Simple Feedback } \\
\text { (High prior) }\end{array}$ & $\mathrm{N}$ & 0.07 \\
\hline $\begin{array}{l}\text { Meyer, B. J. F., Wijekumar, K. K., \& Lin, Y.-C. (2011). Individualizing a } \\
\text { web-based structure strategy intervention for fifth graders' } \\
\text { comprehension of nonfiction. [Feature]. Journal of Educational } \\
\text { Psychology, 103(1), 140-168. }\end{array}$ & $\begin{array}{l}\text { Simple Feedback + Adaptive } \\
\text { Problems v. Simple Feedback } \\
\text { (Middle prior) }\end{array}$ & $\mathrm{N}$ & 0.04 \\
\hline $\begin{array}{l}\text { Gordijn, J., \& Nijhof, W. J. (2002). Effects of complex feedback on } \\
\text { computer-assisted modular instruction. [Feature]. Computers \& } \\
\text { Education, 39(2), 183-200. }\end{array}$ & $\begin{array}{l}\text { Correct Response Feedback + } \\
\text { Guidance + } 2 \text { Additional } \\
\text { Chances to Try Again v. } \\
\text { Correct Response Feedback } \\
\end{array}$ & $\mathrm{N}$ & 0.01 \\
\hline $\begin{array}{l}\text { Meyer, B. J., Wijekumar, K., Middlemiss, W., Higley, K., Lei, P. W., } \\
\text { Meier, C., \& Spielvogel, J. (2010). Web-based tutoring of the structure } \\
\text { strategy with or without elaborated feedback or choice for fifth-and } \\
\text { seventh-grade readers. Reading Research Quarterly, 45(1), 62-92 }\end{array}$ & $\begin{array}{l}\text { Elaborated v. Simple } \\
\text { Computer Feedback (High } \\
\text { Prior) }\end{array}$ & $\mathrm{N}$ & 0 \\
\hline $\begin{array}{l}\text { Walker, E., N. Rummel, et al. (2011). Designing automated adaptive } \\
\text { support to improve student helping behaviors in a peer tutoring } \\
\text { activity. International Journal of Computer-Supported Collaborative } \\
\text { Learning 6(2), 279-306. }\end{array}$ & $\begin{array}{l}\text { Adaptive Feedback for Peer } \\
\text { (plus Cog Tutor) v. Fixed Hints } \\
\text { on Demand (plus Cog Tutor) }\end{array}$ & $\mathrm{N}$ & -0.03 \\
\hline $\begin{array}{l}\text { Walker, E., Rummel, N., \& Koedinger, K. (2009). CTRL: A research } \\
\text { framework for providing adaptive collaborative learning support. User } \\
\text { Modeling and User-Adapted Interaction. The Journal of } \\
\text { Personalization Research (UMUAI), 19(5), 387-431. }\end{array}$ & $\begin{array}{l}\text { Adaptive Feedback for Peer } \\
\text { (plus Cog Tutor) v. Fixed Hints } \\
\text { on Demand for Peer (plus } \\
\text { Cog Tutor) }\end{array}$ & $\mathrm{N}$ & -0.03 \\
\hline $\begin{array}{l}\text { Razzaq, L. \& Heffernan, N. (2009). To tutor or not to tutor: That is the } \\
\text { question. In Dimitrova, Mizoguchi, du Boulay \& Graesser (Eds.) } \\
\text { Proceedings of the } 2009 \text { Artificial Intelligence in Education } \\
\text { Conference. pp. 457-464. ISO Press, Netherlands. }\end{array}$ & $\begin{array}{l}\text { Step by Step Guidance v. } \\
\text { Worked Our Right Answer } \\
\text { Feedback (high prior) }\end{array}$ & $\begin{array}{l}\text { S [in } \\
\text { favor of } \\
\text { control] }\end{array}$ & -0.27 \\
\hline $\begin{array}{l}\text { Dresel, M. (2005). Enhancing academic self-concepts and implicit } \\
\text { theories through computer based attributional feedback. Paper } \\
\text { presented at annual meeting of American Educational Research } \\
\text { Association in Montreal, Canada. }\end{array}$ & $\begin{array}{l}\text { Adaptive Effort-Ability } \\
\text { Feedback + Adaptive Ability- } \\
\text { Effort Feedback v. } \\
\text { Effort/Ability Randomized }\end{array}$ & $\begin{array}{l}\text { S [in } \\
\text { favor of } \\
\text { control] }\end{array}$ & -0.33 \\
\hline
\end{tabular}

\subsection{Calculation and Analysis of Effect Sizes}

The publications we retrieved frequently contained multiple comparison studies. For the purposes of our meta-analysis, we extracted individual effect sizes from each identified independent sample. To obtain effect sizes, we used Cohen's $d$ in the modified form of 
Hedges' g. Calculating Hedges' g involved dividing by the difference of the means between two experimental groups by the pooled standard deviation (Hedges \& Olkin, 1985). We weighted the effect sizes by the inverse variance in order to reduce the bias that may result from the studies' diverse sample sizes. For studies that reported only t- or F-values, we used other data provided to manually calculate effect sizes (Lipsey \& Wilson, 2001).

Our unit of analysis of the effect sizes was based on an independent sample (Wilson, 2009). For example, if a study reported results from two independent experiments, we calculated and listed two effect sizes for that study. Many studies reported separate outcomes for each different form of assessment (e.g., immediate and delayed post tests) of the same study participants. In these cases we generated independent groups by combining the effects of multiple assessment points using the method of Borenstein et al., (2009). The method requires an estimation of the Pearson correlations between distinct outcomes. We estimated the Pearson correlation between distinct outcomes to be 0.3 based on the typical correlations among assessments within our research (Liu et al., 2011).

For each analysis, we employed a weighted random-effects model to calculate estimates of mean effect sizes and 95\% confidence intervals around the estimates, using the software program Comprehensive Meta-Analysis (Borenstein, Hedges, Higgins, \& Rothstein, 2005). Random effects models for statistical significance of mean effect sizes and moderator relationships have the appropriate Type 1 error rate when both the population parameters vary or are the same across studies. In comparison, the fixed effects models have higher Type 1 error rates when the population parameters vary across studies (Hunter \& Schmidt, 2004). The random-effects models generate more conservative results and have been recommended and used by several researchers (e.g., Edmonds, Vaughn, Wexler, Reutebuch, Cable, Tackett, et al., 2009; Ginns, 2005; Hoffler \& Leutner, 2007).

We used a mixed-effects model to perform the moderator analyses as recommended by Borenstein et al. (2009), also using Comprehensive Meta-Analysis. We calculated Q values to indicate whether the differences among groups of the moderator variables reach statistical significance at an alpha level of .05 (Lipsey \& Wilson, 2001). Where moderator effects reached significance and there were more than two groups, we conducted a subsequent analysis to determine which specific group or groups were responsible for the significant outcome (i.e., significantly different from the other groups). Our interpretation of the effect sizes follows Cohen's (1988) guidelines: An effect size of 0.2 is small; 0.5 is moderate; and 0.8 or higher is large.

\section{Results}

Our findings are organized by our two research questions regarding (1) the value of automated adaptive guidance vs. typical instruction, and (2) the value of automated enhanced vs. simple guidance. For each analysis, we first describe the overall effects of automated guidance, present significant moderating variables, and then discuss the 
significant moderating variables relative to the design of guidance to strengthen $\mathrm{K}-12$ student learning.

\subsection{Automated, Adaptive Guidance vs. Typical Instruction}

Overall, results suggest automated guidance can add value to typical, K-12 classroom instruction. After combining assessment outcome categories (generate with selection task; immediate with delayed outcomes), effect sizes ranged from -0.29 to +.97 and were heterogeneous $(\mathrm{Q}=67.96, \mathrm{p}<0.001)$. The mean overall effect size was significant in favor of instruction with automated guidance over typical instruction $(\mathrm{g}=+0.34,95 \% \mathrm{CI}$ $=+0.23$ to $+0.45, \mathrm{p}<.001)$.

Table 4. Overall Effect Size in Automated Guidance vs. Typical Instruction Comparison Studies

\begin{tabular}{ccccccccccc}
\hline & & & $95 \%$ CI & Null test & \multicolumn{2}{c}{ Heterogeneity } \\
\hline Outcome measures & N effects & $\mathrm{g}$ & low & high & $\mathrm{Z}$ & $\mathrm{p}$ & $\mathrm{Q}$ & $\mathrm{p}$ \\
\hline All (auto-typical) & 24 & 0.34 & 0.23 & 0.45 & 6.159 & $<.001$ & 67.965 & $<.001$ \\
\hline
\end{tabular}

Automated guidance demonstrated a significant advantage over each of the activities used by researchers to represent "typical classroom instruction". There was no significant difference among the different typical instructional conditions (worksheets with no teacher guidance $\mathrm{n}=3, \mathrm{~g}=.44$; regular teacher-facilitated instruction $\mathrm{n}=14, \mathrm{~g}=.37$; same computer program in treatment but with non-adaptive guidance or teacher-generated $n=7$, $\mathrm{g}=.25)$.

The studies of automated guidance were conducted largely in upper elementary and middle school classrooms (Gr. 5-8, 85\%, 9-12, 15\%), in the areas of mathematics and science (math $44 \%$, science $37 \%$, language arts or social studies, 19\%). Automated guidance accompanied a range of generative item types, as well as selection tasks $(33 \%$ written explanations or essays, $19 \%$ stamp drawings or concept diagrams, $26 \%$ math operations or formula, $19 \%$ selection tasks). The goals of the guidance varied from enhancing conceptual understanding (56\%), to diagnosing errors (33\%), to promoting self-monitoring skills (4\%). The majority of the studies used a pre-posttest design to measure learning gains (61\%) whereas others also drew on embedded assessments (13\%), a delayed posttest $(13 \%)$ or a combination of measures $(13 \%)$.

\subsubsection{Significant moderators of automated guidance vs. typical instruction}

We analyzed potential moderator variables across studies that compared instruction with automated guidance to typical instruction. Results revealed that only one of the thirteen moderators, prior knowledge, yielded significant differences between experimental groups (Table 5). Prior knowledge was determined by student performance on the pretest, or on their initial response to the task with automated guidance. We found instruction with automated guidance to be significantly more effective than typical instruction for 
students with low $(\mathrm{g}=.59)$ or medium $(\mathrm{g}=.35)$ prior knowledge of the topic compared to students who began with high prior knowledge $(\mathrm{g}=-.14)$. A subsequent analysis indicated that the high prior knowledge students were significantly different from the low and medium prior knowledge students combined in terms of their interactions with the automated guidance $(\mathrm{p}=.006)$. High prior knowledge students tended to benefit more from typical instruction than from automated guidance.

Table 5. Significant Moderator Variables in Automated Guidance vs. Typical Instruction Comparison Studies

\begin{tabular}{lllllll}
\hline Significant Moderator Variables $(p)$ & $N$ & $g$ & $S E$ & $95 \%$ CI low & $95 \%$ CI high & p-value between groups \\
\hline Prior Knowledge** & & & & & & \\
\hline Low & 5. & +0.59 & 0.10 & 0.40 & 0.79 & .002 \\
\hline Mixed/medium & 15. & +0.35 & 0.06 & 0.24 & 0.46 & \\
\hline High & 4. & -0.14 & 0.19 & -0.50 & 0.22 \\
\hline a**=p $<.005$ & & & & &
\end{tabular}

\subsubsection{Non-significant moderators of automated guidance vs. typical instruction}

We computed separate mean effect sizes for the selection and generation learning outcomes on the embedded, pre and posttest, and delayed posttest results. None of the differences between these outcomes were significant. Additionally, none of the other moderators [grade level, domain, item type, kind of automated guidance, adaptive problems, type of assessment on which student received guidance, duration, or type of scoring technology] showed significant differences. This result suggests that multiple forms of automated guidance appear to be useful across different K-12 classroom contexts.

\subsubsection{Prior knowledge and automated guidance}

The advantage of automated guidance relative to typical instruction for low and medium/ mixed prior knowledge students is consistent with findings from prior reviews of guidance (Black \& Wiliam, 1998). This suggests that most students need more guidance to master the content than they are able to receive in a typical classroom and that these students are eager to use such guidance when it is made available. When the automated guidance was available upon student request, low performing students took greater advantage of its help features (e.g., more frequent request for detailed hints) than did high performing students (Beal, Arroyo, Cohen \& Woolf, 2010; Beal, Walles, Arroyo, \& Woolf, 2007). Indeed, studies by Beal, Arroyo, et al. and Beal, Walles, et al. show greater use of automated guidance to be significantly correlated with higher learning gains.

Students with high prior knowledge made greater gains when receiving guidance from their teacher than when receiving automated guidance. In the three studies reporting negative effect sizes for high prior knowledge students, the automated guidance was 
compared to "normal" teacher-led instruction as opposed to typical instruction defined by a worksheet or computer program with no guidance at all (Beal et al., 2010, Beal et al., 2007; McElhaney et al., 2012). Little if any information was provided in the papers to characterize the type of guidance provided in "normal" teacher-led instruction. The teacher's instruction may have been more effective for high prior knowledge students given that by virtue of being high prior knowledge they have already demonstrated success in learning from the typical instructional context. The automated guidance alternatively presented a new form of instruction that high prior knowledge students have not previously mastered.

The high prior knowledge students chose to ignore the automated guidance (Beal et al., 2010, Beal et al., 2007) or perhaps perceived it as extraneous or unhelpful (McElhaney et al., 2012). In the studies by Beal, Arroyo, et al. and Beal, Walles, et al. the automated guidance was available to students on demand by pressing a help button. Students with high prior knowledge were the least likely of all students in both studies to request help, despite having made errors on the mathematics problems. In the study by McElhaney et al. (2012), the teachers provided more concise and general guidance than the detailed automated guidance. The concise guidance, authors hypothesize, may have targeted a smaller knowledge gap and hence nudged the low prior knowledge students further in the right direction, whereas the detailed automated guidance may have been seen as distracting or unnecessary by the high prior knowledge students. This resonates with previous research suggesting that reducing guidance may bring about a "desirable difficulty" for high prior knowledge students (Bjork \& Linn, 2006). Providing less detailed guidance may prompt students to slow down their learning process and in doing so enhance long term learning outcomes.

While researchers hypothesized that typical teacher instruction was more beneficial for high prior knowledge students, few studies actually addressed how teachers interacted with the automated guidance in the classroom. The few studies that found promising outcomes concerned how the teacher and computer worked together (Koedinger et al., 2010; McElhaney et al., 2012; White et al., 2010). For example, Koedinger et al., (2010) found automated, adaptive guidance may have helped teachers lead large classes with diverse students. The ASSISTments program provided students with detailed individualized guidance in solving mathematics problems and provided teachers with diagnostic reports of their students' performance. In this study, the fraction of Special Education students in mainstream classrooms significantly increased over three years, while in the control schools the fraction of Special Education students in the classes remained constant. Teachers reported that ASSISTments provided sufficient assistance for many of the students in the class, making it possible for the teacher to help the students with Special Education needs.

In summary, these results show that automated guidance adds value to typical instruction for most students. Studies specifically highlight the advantages of automated guidance for students who are initially low or moderately performing. This outcome draws attention to the difficulties teachers face when guiding large classes, attempting to customize instruction to diverse learners, and teaching difficult concepts where many students are 
likely to struggle. Further research is needed on what kinds of automated guidance are needed to improve outcomes for diverse students.

\subsection{Enhanced vs. Simple Automated Guidance}

Our first analysis showed that automated, adaptive guidance is valuable for K-12 contexts. To answer our second research question (what kinds of automated, adaptive guidance are most effective), we compared enhanced and simple guidance designs. After combining assessment outcome categories (generation with selection task; immediate with delayed outcomes), effect sizes ranged from -0.33 to +1.59 and were heterogeneous $(\mathrm{Q}=80.60, \mathrm{p}<0.001)$. The mean overall effect size was significant in favor of the enhanced automated guidance over simple automated guidance $(\mathrm{g}=+0.27,95 \% \mathrm{CI}=$ +0.15 to $+0.38, \mathrm{p}<.001)$.

In each study, enhanced guidance in the treatment condition was intended to promote conceptual advancement, strengthen the coherence of student writing, or facilitate selfmonitoring. The form of the enhanced guidance was consistently text-based, and in most cases integrated into a curriculum unit. Meanwhile simple guidance in the control condition included providing the correct answer $(n=22 \%)$, detecting errors $(n=30 \%)$, giving direct instructions for improvement (without encouraging self-monitoring) $(\mathrm{n}=19 \%)$, and giving hints and/or assigning non-adaptive problems $(\mathrm{n}=30 \%)$.

Overall, results support the value of enhanced guidance that encourages students to reconsider their ideas, rather than simpler guidance that tells students the correct answer. Enhanced automated guidance provided contextualized hints for conceptual advancement or to improve students' self-monitoring strategies, as opposed to simple guidance, which most often verified the correctness of a student response and/or provided the student with the correct answer.

Table 6. Overall effect size in enhanced vs. simple automated guidance comparison studies

\begin{tabular}{lcccccccccc}
\hline & & & & & & & & \\
& & & & & Null test & & Heterogeneity \\
\hline Outcome measures & N effects & $\mathrm{g}$ & low & high & $\mathrm{Z}$ & $\mathrm{p}$ & $\mathrm{Q}$ & $\mathrm{p}$ \\
\hline All (auto enhanced-auto simple) & 29 & +0.27 & 0.15 & 0.38 & 4.37 & $<.001$ & 80.60 & $<.001$ \\
\hline
\end{tabular}

Similar to the first analysis (automated, adaptive guidance vs. typical instruction), the majority of studies of enhanced v. simple automated guidance were conducted in upper elementary and middle school classrooms $(71 \%)$ rather than high school $(29 \%)$. Unlike the first analysis however, there was an even distribution of studies across the domains of mathematics (39\%), science (32\%) and the language arts (29\%). Further, the majority of studies used pre and posttest measures of student learning in addition to embedded assessments and delayed posttests. Outcomes measured students' conceptual knowledge gains as well as self-monitoring strategies and writing skills. 


\subsubsection{Significant moderators of enhanced vs. simple automated guidance}

Consistent with findings from our analysis of automated guidance vs. typical instruction, enhanced automated guidance showed a greater advantage over simple guidance, particularly for students with limited prior knowledge in the target topic. The moderator analysis (Table 7) suggests features of automated guidance that significantly strengthened learning outcomes. These features include promoting self-monitoring (e.g. improving student strategy for distinguishing ideas), scaffolding students' work during generation tasks that encourage linking ideas (e.g., creating concept diagrams) as opposed to during selection tasks (e.g., multiple-choice questions), and measuring learning using generation as opposed to selection tasks.

Table 7. Significant moderator variables in Enhanced vs. Simple Guidance comparison studies

\begin{tabular}{|c|c|c|c|c|c|c|}
\hline Significant Moderator Variables ( $p$ ) & $N$ & $g$ & $S E$ & $\begin{array}{l}95 \% C I \\
\text { low }\end{array}$ & $\begin{array}{l}95 \% C I \\
\text { high }\end{array}$ & $\begin{array}{l}p \text { value betweer } \\
\text { groups }\end{array}$ \\
\hline \multicolumn{7}{|l|}{ Encouraged Self-Monitoring** } \\
\hline Yes & 11 & 0.50 & 0.12 & 0.26 & 0.74 & 0.001 \\
\hline No & 17 & 0.17 & 0.06 & 0.06 & 0.28 & \\
\hline \multicolumn{7}{|l|}{$\begin{array}{l}\text { Learning Activity on which Student } \\
\text { Gets Guidance* }\end{array}$} \\
\hline Drawing with stamps or concept diagram & 5 & 0.72 & 0.19 & 0.34 & 1.10 & .017 \\
\hline Written explanation & 4 & 0.27 & 0.15 & -0.02 & 0.57 & \\
\hline Mathematical operations or formulas & 14 & 0.22 & 0.07 & 0.07 & 0.37 & \\
\hline Selection & 6 & 0.06 & 0.08 & -0.10 & 0.23 & \\
\hline \multicolumn{7}{|l|}{ Prior Knowledge* } \\
\hline Low & 5 & 0.32 & 0.17 & -0.02 & 0.66 & 0.023 \\
\hline Mixed or medium & 20 & 0.32 & 0.07 & 0.17 & 0.46 & \\
\hline High & 4 & -0.02 & 0.1 & -0.22 & 0.17 & \\
\hline \multicolumn{7}{|l|}{ Outcome measure* } \\
\hline Generate task & 12 & 0.43 & 0.13 & 0.17 & 0.68 & 0.020 \\
\hline Selection task & 7 & 0.11 & 0.08 & -0.03 & 0.26 & \\
\hline Mixed & 9 & 0.25 & 0.08 & 0.09 & 0.40 & \\
\hline Not specified & 1 & 0.01 & 0.07 & -0.14 & 0.16 & \\
\hline
\end{tabular}


$\mathrm{a} * *=\mathrm{p}<.005 ; * \mathrm{p}=<.05$

We conducted subsequent analyses for the significant moderators with more than two categories to distinguish which specific group was significantly different from the others.

Prior knowledge. High prior knowledge students were significantly different from the low and medium prior knowledge students in terms of their interactions with the enhanced automated guidance, (high v. low and medium prior knowledge combined, $\mathrm{p}=.005$ ). Low and medium prior knowledge students benefitted more from the enhanced automated guidance than the simple automated guidance. High prior knowledge students however performed equally when receiving enhanced or simple automated guidance.

Learning activity in which the student gets guidance. Enhanced automated guidance strengthened students' reasoning on generative activities including drawing and writing, whereas simple automated guidance was sufficient for selection and math problems. Generative activities including drawing, writing and solving math problems with enhanced automated guidance yielded significantly greater learning outcomes than did selection activities with enhanced guidance $(\mathrm{p}=.015)$. Students benefitted the most from enhanced guidance design features in activities that involved creating and revising drawings or concept diagrams as opposed to writing, solving math problems, and selecting from given options $(\mathrm{p}=.007)$. Solving math problems with enhanced automated guidance produced comparable learning outcomes to selection activities with enhanced automated guidance $(\mathrm{p}=.167)$.

Outcome measure. Generative outcome measures (e.g. writing an explanation) produced significantly greater evidence of learning following the enhanced automated guidance compared to selection outcome measures (e.g. multiple-choice) $(\mathrm{p}=.04)$.

\subsubsection{Non-significant moderators}

There was no effect on learning for the moderator variables grade level, domain, kind of automated guidance, adaptive problems, duration, or type of scoring technology. This result suggests that enhanced and simple guidance designs are effective across grades and domains. Further research is needed to identify design features of automated guidance and adaptive problems that will advance student learning outcomes.

\subsubsection{Enhanced automated guidance to support distinguishing and integrating ideas}

The guidance design features that encouraged students to reflect on their understanding and refine their approach to distinguishing ideas, or to generate and strengthen the links among their ideas yielded significantly greater student learning than simple right/wrong forms of automated guidance.

Guidance that promoted self-monitoring was more likely to improve learning outcomes than guidance that only addressed content. This guidance approach encouraged students to stop and reflect on their understanding and distinguish a more successful learning strategy. To trigger the self-monitoring guidance, the software used log data indicators 
such as time on task, frequency of guidance requests, type of guidance requests (right answer vs. detailed hint), and frequency of revisiting pages in the unit that contained evidence about the topic. Examples from the five programs that incorporated selfmonitoring guidance are shown in Table 8.

Self-monitoring guidance coupled with content guidance within the Betty's Brain environment, as shown in Figure 1, demonstrated the greatest effect on learning. The selfmonitoring guidance prompted students to use a broader array of learning strategies including: prompting Betty to explain the causal reasoning between links rather than checking only for the correctness of Betty's concept map; revisiting pages in the unit that included evidence about the target topic before editing Betty's concept map. Students receiving self-monitoring guidance and content guidance made significantly greater gains on the free response and concept map items from the pre to the posttest than students who received only content guidance.

One possible explanation for the large effects in the Betty's Brain studies lies in the unique, overarching learn-by-teaching framework that guided the design of the environment and automated guidance. Consistent with the philosophy of learning by teaching (Bransford et al., 1999), students may be more motivated to respond to selfmonitoring guidance when the goal is to teach a computer-based tutee (i.e., Betty), than when it is to improve their own performance. In order to better determine whether this is the case, future studies might explore how varying the motivational goals of selfmonitoring guidance differentially affects students' learning outcomes.

Table 8. Examples of Automated, Adaptive Guidance to Promote Self-Monitoring

\begin{tabular}{|c|c|c|c|c|c|}
\hline Study & Tool & $\begin{array}{l}\text { Guidance on } \\
\text { Content } \\
\text { [Control } \\
\text { Condition] }\end{array}$ & $\begin{array}{l}\text { Guidance on Self-Monitoring } \\
\text { [Given in addition to the } \\
\text { Content Guidance] }\end{array}$ & $\begin{array}{l}\text { Indicator for } \\
\text { automated } \\
\text { scoring }\end{array}$ & $\begin{array}{l}\text { Effect } \\
\text { Size }\end{array}$ \\
\hline $\begin{array}{l}\text { Leelawong \& } \\
\text { Biswas (2008) }\end{array}$ & $\begin{array}{l}\text { Betty's Brain } \\
\text { concept map }\end{array}$ & $\begin{array}{l}\text { Conceptual and } \\
\text { specific content } \\
\text { guidance }\end{array}$ & $\begin{array}{l}\text { Student checks the correctness } \\
\text { of their concept diagram by } \\
\text { asking Betty a question, but } \\
\text { student does not ask Betty to } \\
\text { explain the causal reasoning } \\
\text { behind her answer. } \\
\text { Betty: "Let's see, you have } \\
\text { asked me a lot of questions, but } \\
\text { you have not asked for my } \\
\text { explanations lately. Please } \\
\text { make me explain my answers } \\
\text { so you will know if I really } \\
\text { understand." }\end{array}$ & $\begin{array}{l}\text { Log file noting } \\
\text { what embedded } \\
\text { resources the } \\
\text { student used } \\
\text { when editing } \\
\text { and checking } \\
\text { concept map; } \\
\text { identification } \\
\text { of guess and } \\
\text { check approach }\end{array}$ & 1.55 \\
\hline $\begin{array}{l}\text { Wade-Stein \& } \\
\text { Kintsch } \\
(2004)\end{array}$ & $\begin{array}{l}\text { Summary } \\
\text { Street long } \\
\text { essays }\end{array}$ & $\begin{array}{l}\text { Detailed } \\
\text { guidance on } \\
\text { content and } \\
\text { grammar of } \\
\text { essay }\end{array}$ & $\begin{array}{l}\text { A dynamic visualization of } \\
\text { student progress illustrating the } \\
\text { degree of improvement with } \\
\text { each revision/feedback cycle on } \\
\text { metrics of content adequacy, } \\
\text { relevance, redundancy, length }\end{array}$ & $\begin{array}{l}\text { Natural } \\
\text { language } \\
\text { processing } \\
\text { analysis of } \\
\text { student essays }\end{array}$ & .76 \\
\hline Rebolledo- & Ecolab & Adaptive & "Be bold and try and take more & Number of & .56 \\
\hline
\end{tabular}




\begin{tabular}{|c|c|c|c|c|c|}
\hline $\begin{array}{l}\text { Mendez, } \\
\text { Luckin, et al. } \\
\text { (2011) }\end{array}$ & concept map & $\begin{array}{l}\text { activities based } \\
\text { on performance }\end{array}$ & $\begin{array}{l}\text { challenge" } \\
\text { "Use less help" }\end{array}$ & $\begin{array}{l}\text { questions } \\
\text { asked; level of } \\
\text { difficulty of } \\
\text { problems } \\
\text { selected }\end{array}$ & \\
\hline $\begin{array}{l}\text { Walker, } \\
\text { Rummel \& } \\
\text { Koedinger } \\
\quad(2011)\end{array}$ & $\begin{array}{l}\text { Cognitive } \\
\text { Tutor - } \\
\text { Adaptive } \\
\text { Peer Tutor }\end{array}$ & $\begin{array}{l}\text { Cognitive Tutor } \\
\text { for solving } \\
\text { algebraic } \\
\text { problems }\end{array}$ & $\begin{array}{l}\text { "Sara, think about the last help } \\
\text { you gave. Why did you say } \\
\text { that? Can you explain more?" } \\
\text { "Sara, when helping, give } \\
\text { examples or facts your partner } \\
\text { already understands" }\end{array}$ & $\begin{array}{l}\text { Natural } \\
\text { language } \\
\text { processing of } \\
\text { peer dialogue }\end{array}$ & .48 \\
\hline $\begin{array}{l}\text { Dresel \& } \\
\text { Haugwitz } \\
(2008)\end{array}$ & $\begin{array}{l}\text { MatheWarp } \\
\text { equations }\end{array}$ & $\begin{array}{l}\text { Right/Wrong } \\
\text { guidance }\end{array}$ & $\begin{array}{l}\text { "This good work can be traced } \\
\text { back to the high level of effort } \\
\text { you gave" } \\
\text { "You have a good grasp of the } \\
\text { topic. If you spend more time } \\
\text { to answer the problems you } \\
\text { may get them all correct this } \\
\text { time." }\end{array}$ & $\begin{array}{l}\text { Time on task; } \\
\text { level of } \\
\text { difficulty of } \\
\text { questions } \\
\text { answered }\end{array}$ & .22 \\
\hline
\end{tabular}

Fig 1. Betty's Brain.

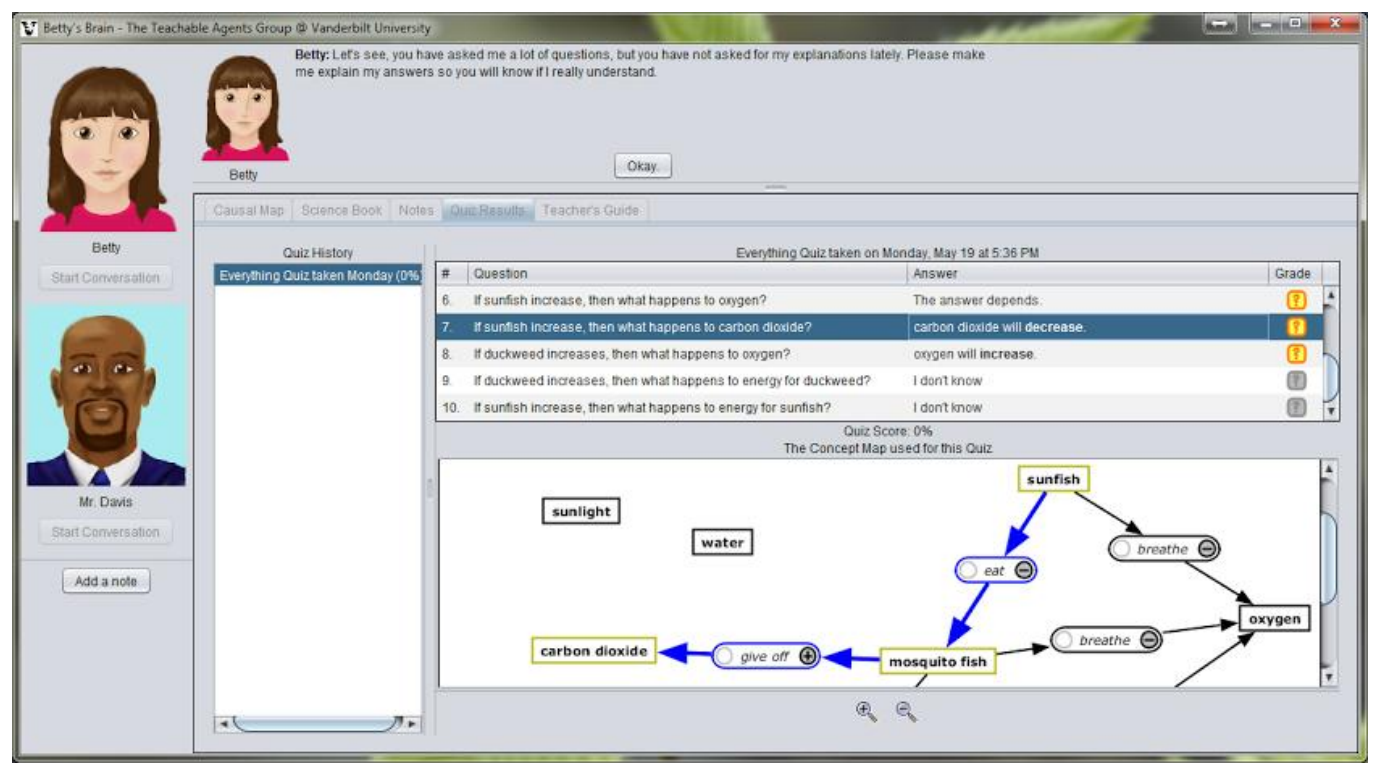

Student generated a concept diagram. Then student asked Betty to take a quiz based on the diagram. After answering 10 questions, Betty responded with the self-monitoring prompt, shown to the right of her image, encouraging the student to ask Betty to provide an explanation for her answers rather than just give an answer.

Studies on the self-monitoring guidance in Summary Street have also shown relatively large effects on student learning (Wade-Stein \& Kintsch, 2004). In addition to offering students detailed guidance on the content and grammatical structure of their essays, Summary Street also structures students' work within cycles of guidance and revision and 
displays students' improvement over time (Fig 2). Whereas guidance on content and grammar should have value on its own, it may be especially effective when the learning environment promotes a habit of meaningful revision, thus encouraging students to attend to that guidance.

Fig 2. Summary Street

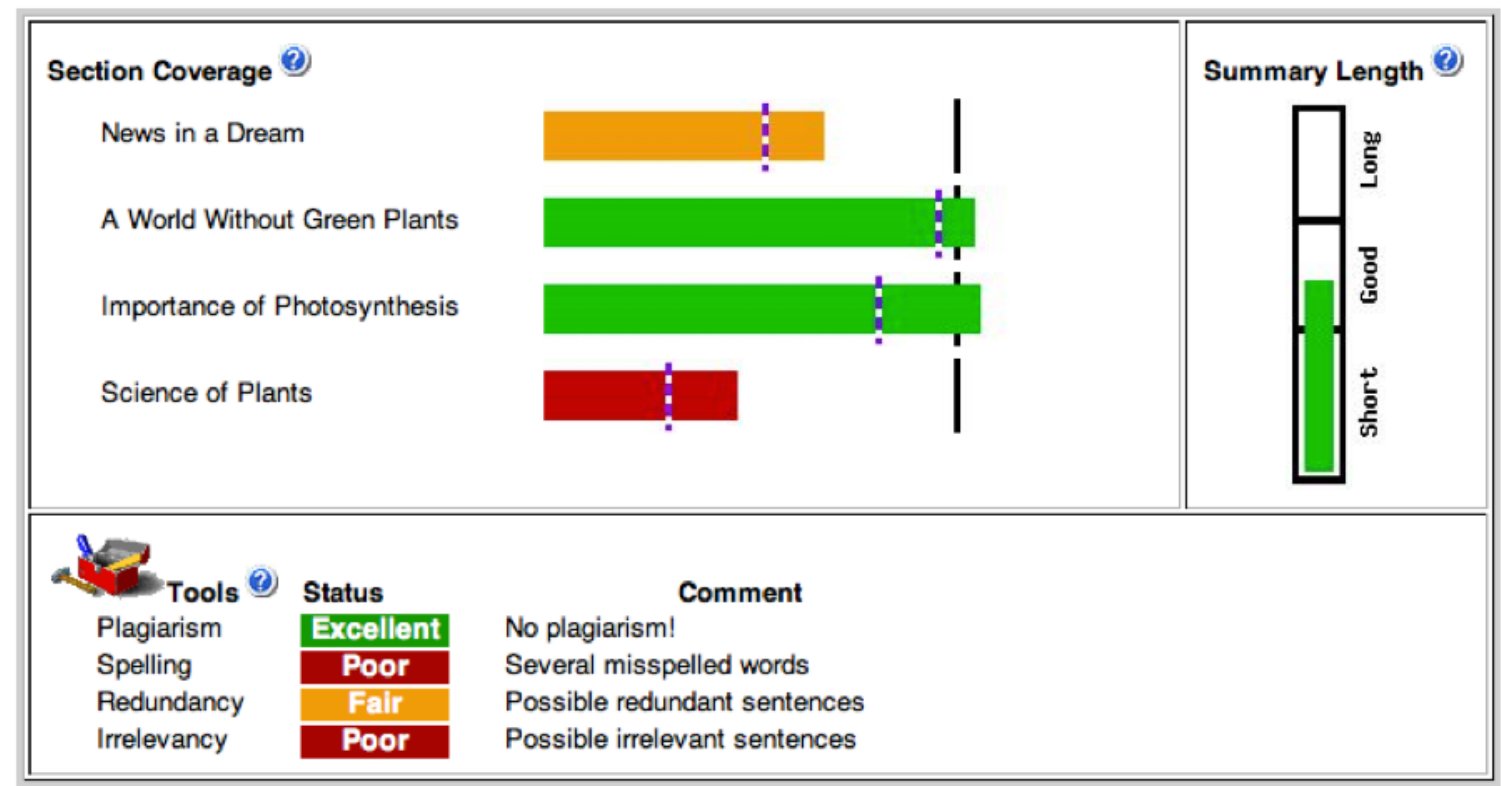

Student generated a summary of an essay, and then revised their summary based on the Summary Street guidance. Each row is a different paragraph. The colored bars demonstrate performance (e.g orange=fair), and the dashed line marks performance on the initial summary so the student can see their improvement with each revision.

[Reference: Kintsch, W., \& Kintsch, E. (2012). LSA in the classroom. In P. M McCarthy \& C. Boonthum-Denecke (Eds.): Applied natural language processing: Identification, investigation, and resolution (pp. 158-168). Hershey, PA: IGI Global. p. 5.]

The large effects of self-monitoring guidance across environments may be explained using several perspectives on learning. Simple reminders to slow down and reflect may be especially helpful during student-paced learning activities, where teacher direction is not consistently present. Student-paced activities are typical of technology-enhanced environments. From a classroom culture perspective, self-monitoring guidance, embedded within a pattern of guidance and revision, may create classroom norms in where students expect and respond to other kinds of guidance, and are therefore more likely to use it effectively. From a cognitive perspective, self-monitoring guidance may encourage a kind of thinking that is crucial for deep understanding. Indeed, the selfmonitoring guidance across studies encouraged students to distinguish among new and existing information, either by having students revisit evidence in the unit, and/or by having students ask themselves questions about their learning strategies. Although guidance that did not include self-monitoring prompts still allowed students to encounter similar ideas to their peers in the treatment conditions, it did not always push students to reconcile specific new information with their initial views.

Enhanced automated guidance had greater effects on learning gains with assessment items requiring students to generate and strengthen the links among their ideas, than with items asking students to select an answer from a list. Students receiving guidance for 
their writings, drawings, or application of mathematical formulas made significantly greater learning gains than students receiving guidance for selections from multiplechoices. Relative to simple automated guidance, enhanced automated guidance was most effective for student generated concept maps and drawings, compared to all other item types.

The advantage for guidance on student drawings and concept maps relative to other tasks may reflect the current state of automated scoring technologies. Scoring of concept maps and stamp drawings, in which all elements are predetermined (e.g., nodes, links, stamp identities), is based on relations, locations and proximities of objects. This means that automated scores can be more precise, allowing instructional designers to construct associated automated guidance that is better aligned with students' knowledge. In contrast, automated scoring of open-ended items, such as essays, is based on natural language processing technologies that are still undergoing improvement. There is more uncertainty in the scoring and, therefore, in the alignment of the targeted guidance with the students' knowledge. Likewise improvement of essays is influenced by both student writing ability and content knowledge, while concept maps and stamp drawings are more likely to be influenced by content knowledge alone.

In spite of the apparent constraints of concept maps and stamp drawings relative to freeform written responses, these appear to be remarkably expressive response forms, and particularly efficient at eliciting students' ideas. Compared to selection tasks, such as multiple-choice questions, concept maps and stamp drawings allow for the identification of particular links among a student's ideas, facilitating the association of targeted guidance with those ideas. For example, Ryoo \& Linn (2014) constructed a concept diagramming task in which students received either a prompt that targeted a specific missing or non-normative idea in students' diagrams, or a prompt that targeted a general missing concept and encouraged students to revisit a related piece of evidence from earlier in the unit. (An example of a specific prompt is You are going in a good direction! Now improve your diagram to show that plants use energy stored in glucose during cellular respiration, while an example of a conceptual prompt is You are going in a good direction! Now review the visualization in Step 3.9 to find out how plants get energy during cellular respiration and improve your diagram). The conceptual prompt with encouragement to revisit was significantly more effective than the specific prompt. Students reconsidered links among their ideas and strengthened their concept diagrams as a whole, as opposed to those students receiving the specific guidance that reconsidered a discrete idea and subsequently modified only a single icon, which in most cases did not improve their diagram.

We computed separate mean effect sizes for the selection and generative learning outcome measures and the embedded, pre- post-test, delayed post test outcomes. We found a negligible mean effect of the selection tasks $(g=0.11)$, which suggests that enhanced automated guidance offers little advantage to learners for helping them simply recall information. We found a significant positive mean effect for the generate outcomes ( $\mathrm{g}=+0.43,95 \% \mathrm{CI}=+0.08$ to +0.48 ), illustrating that enhanced automated guidance appears to offer a measurable advantage for conceptual learning. 


\subsubsection{Prior knowledge and enhanced automated guidance}

Enhanced automated guidance was significantly more effective for students with limited prior knowledge of the target topic. This result is consistent with those of our first analysis (automated guidance vs. typical instruction). Razzaq \& Heffernan's (2009) comparison study of enhanced v. simple guidance (enhanced=providing detailed step-bystep guidance; simple= worked out solution) indicated potential in varying the form of guidance based on levels of prior knowledge in order to enhance learning outcomes of all students. They found that initially proficient students could take advantage of annotated solutions designed by an expert instructor to improve their performance on subsequent mathematics problems. Initially less proficient students meanwhile benefited from guidance that emulated an expert teacher by asking questions to prompt students to reflect rather than directly offering correct solutions. This findings suggests that high prior knowledge students may require forms of guidance that are highly distinct from guidance needed by other learners. Identifying what forms of guidance are most effective for specific types of learners merits further research.

\section{Limitations}

This research is limited by the number of studies that are available that investigate the effects of automated guidance on K-12 student learning. There are numerous studies investigating the validity of automated scoring technologies (we identified 30 papers describing new scoring technologies in our search), but they remain untested in K-12 settings. The paucity of studies poses limitations that readers should bear in mind when interpreting our findings.

Second, the studies included in our meta-analysis varied widely in the nature of their control conditions and the instructional contexts in which the guidance was studied. In order to collect a large enough number of studies to produce interpretable results, we had to collapse potentially independent moderator variables into broader categories for analysis. Consequently, we are unable to provide a more fine-grained interpretation of the findings than our current categories permit.

\section{Future Questions}

This study helps synthesize current insights regarding designs of automated guidance that are most effective in which instructional settings and for which types of students. Our study also suggests several avenues for future research and development that would deepen the educational research community's understanding of how technology can benefit learning and instruction. The questions raised by this meta-analysis suggest several clear directions for future research.

1. What kinds of self-monitoring guidance are most effective? Although we found selfmonitoring guidance to have large effects on student learning, we could not separate the effects of different kinds of self-monitoring guidance. Specifically, for the purposes of our analysis, we considered self-monitoring guidance to include the provision of student progress-monitoring displays (Wade-Stein \& Kintsch, 2004), encouragement to make 
greater or less use of on-demand hints (Rebolledo-Mendez, et al. 2011), prompts for students to elaborate on given explanations (Walker, Rummel \& Koedinger, 2011) or to ask a computer tutee to elaborate (Leelawong \& Biswam, 2008), and suggestions to put in more time and effort on subsequent attempts at similar problems (Dresel \& Haugwitz, 2008). To further distinguish what features and conditions make self-monitoring guidance most effective, further studies are needed that focus on the impacts of different kinds of self-monitoring guidance on students' learning. Designers may take advantage of rich logged data to investigate nuanced self-monitoring practices, such as revisiting prior evidence (Ryoo \& Linn, 2014), goal selection (Azevedo, Moos, Johnson \& Chauncey, 2010), and changing variable settings to iterate on the design of experiments within simulation environments (San Pedro et al., 2013).

2. How can guidance be made valuable to students of all levels of knowledge and ability? One of the greatest potential benefits of automated scoring systems is that more students will benefit from guidance customized to their personal needs and abilities. Studies included in our analysis have examined the differential impacts of automated guidance on students with regard only to different levels of prior knowledge, and found such guidance to be most effective for students with low prior knowledge. This finding is potentially due to a ceiling effect: high performing students may have already grasped the key concepts in an item, and thus found no suggestions in the given guidance for further improvement. At the same time, other studies (e.g., Razzaq \& Heffernan, 2009) suggest that high performing students $d o$ still benefit from guidance, albeit from guidance of a different kind than what low performing students receive. Thus, a question for future research is, what kinds of guidance benefit high vs. low performing students, or students from otherwise different starting points or trajectories in their understanding? How might automated guidance be tailored to assist already high performing students to move further beyond the expectations of the lesson and toward achieving higher personal goals?

\section{How might automated guidance best be integrated into teachers' practices? Teachers} play critical roles in ensuring the effectiveness of the automated guidance. Studies report that teachers appreciate the ways these systems can supplement their instruction, especially when they have little time to provide the needed individualized guidance to their increasingly large classrooms of students. When guidance is automated teachers can direct their efforts to students the guidance system identifies as not making progress (Poulsen, Hastings, \& Allbritton, 2007; Rich, 2012). Teachers can also review automated scores to gauge overall class progress or to design activities that address specific conceptual difficulties (Koedinger et al., 2010; McElhaney et al., 2012;). Instructors can use student responses immediately to adjust their instruction and annually to revise instruction for the next group of students. Finding ways to integrate automated guidance into existing instruction would allow teachers more time to assist individual students in need and result in greater, more wide-ranging impacts on student learning. These issues raise the critical question: how can automated scoring and guidance tools best be design to be used as a teaching partner?

\subsection{Conclusions}


In our meta-analysis, the features of automated guidance that had the greatest positive effects on students' learning outcomes were also those that emulated the guidance practices of expert teachers. Successful teachers, as described by Leinhardt \& Steele (2005), "move figuratively and literally to the side of the discussion." This statement does not indicate that the teacher should become invisible or move into the background, but rather that the teacher makes space for students' ideas to become visible and to be interrogated. Effective teachers elicit students' ideas, use these to diagnose their reasoning and to choose a productive learning path, and continue to help students monitor their understanding with metacognitive questions. This approach is well documented by researchers as an effective way to strengthen students' learning, but one that is extremely difficult even for experienced teachers to master. Automated guidance that can effectively support teachers in these roles, especially amid the growing demands on teachers' time and attention, is where we believe the greatest promise for automated scoring and guidance technologies lies.

\section{Acknowledgements}

This material is based upon work supported in part by the National Science Foundation under Grant DRL-1119670: Continuous Learning and Automated Scoring in Science (CLASS); DRL-0918743: Visualizing to Integrate Science Understanding for All Learners (VISUAL); and Cumulative Learning using Embedded Assessment Results (CLEAR) DRL-0822388. Any opinions, findings, and conclusions or recommendations expressed in this material are those of the author(s) and do not necessarily reflect the views of the National Science Foundation. 
References [* papers are included in the meta-analyses]

*Aleven, V. A., \& Koedinger, K. R. (2002). An effective metacognitive strategy: Learning by doing and explaining with a computer-based cognitive Tutor. Cognitive Science, 26, 147-179.

Anderson, J. R., Corbett, A. T., Koedinger, K. R., \& Pelletier, R. (1995). Cognitive Tutors: Lessons learned. Journal of the Learning Sciences, 4(2), 167-207.

Arnott, E., Hastings, P., \& Allbritton, D. (2008). Research methods tutor: Evaluation of a dialogue based tutoring system in the classroom. Behavioral Research Methods 40(3), 694-698.

Azevedo, R. (2005). Computers environments as metacognitive tools for enhancing learning. Educational Psychologist, 40(4), 193-197.

Azevedo, R., Moos, D. C., Johnson, A. M., \& Chauncey, A. D. (2010). Measuring cognitive and metacognitive regulatory processes during hypermedia learning: Issues and challenges. Educational Psychologist, 45(4), 210-223.

Baker, R., Walonoski, J., Heffernan, N., Roll, I., Corbett, A., \& Koedinger, K. R. (2008). Why students engage in "gaming the system" behavior in interactive learning environments. Interactive Learning Research, 19(2), 185-224.

*Beal, C. R., Arroyo, I., Cohen, P. R., \& Woolf, B. P. (2010). Evaluation of AnimalWatch: An intelligent tutoring system for arithmetic and fractions. Journal of Interactive Online Learning, 9(1), 64-77.

*Beal, C. R., Walles, R., Arroyo, I., \& Woolf, B. P. (2007). On-line tutoring for math achievement testing: A controlled evaluation. Journal of Interactive Online Learning, 6(1), 43-55.

Bjork, R. A. (1994). Memory and metamemory considerations in the training of human beings. In Metacognition: Knowing about Knowing. J. Metcalfe and A. Shimamura. Cambridge, MA, MIT Press: 185-205.

Bjork, R., \& Linn, M.C. (2006). The science of learning and the learning of science, APS Observer, 19(3), 1-2.

Black, P., \& Wiliam, D. (1998). Inside the black box: Raising standards through classroom assessment. Phi Delta Kappan, 80(2), 139-144.

Borenstein, M., Hedges, L., Higgins, J., \& Rothstein, H. (2005). Comprehensive metaanalysis version 2. Englewood, NJ: Biostat.

Borenstein, M., Hedges, L. V., Higgins, J. P. T., \& Rothstein, H. R. (2009). Introduction to meta-analysis. Chichester, UK: Wiley

Bransford, J. D., Brown, A. L., \& Cocking, R. R. (1999). How people learn: Brain, mind, experience, and school. Washington, DC: National Academy Press.

*Chin, D. B., Dohmen, I. M., Cheng, B. H., Oppezzo, M. A., Chase, C. C., \& Schwartz, D. L. (2010). Preparing students for future learning with Teachable Agents. Educational Technology Research and Development, 58(6), 649-669.

*Dresel, M. (2005). Enhancing academic self-concepts and implicit theories through computer based attributional feedback. Paper presented at the American Educational Research Association Annual Conference, Montreal, Canada.

*Dresel, M., \& Haugwitz, M. (2008). A computer-based approach to fostering motivation and self-regulated learning. The Journal of Experimental Education, 77(1), 3-18. doi: 10.3200/jexe.77.1.3-20. 
Edmonds, M. S., Vaughn, S., Wexler, J., Reutebuch, C., Cable, A., Tackett, K. K., et al. (2009). A synthesis of reading interventions and effects on reading comprehension outcomes for older struggling readers. Review of Educational Research, 79(1), 262-300.

*Franzke, M., Kintsch, E., Caccamise, D., Johnson, N., \& Dooley, S. (2005). Summary Street: Computer support for comprehension and writing. Journal of Educational Computing Research, 33(1), 53-80.

Furtak, E. M., Seidel, T., Iverson, H., \& Briggs, D. C. (2012). Experimental and quasiexperimental studies of inquiry-based science teaching: A meta-analysis. Review of Educational Research, 82, 300-329.

*Gerard, L. F., Linn, M. C., \& Liu, O. L. (2012). How well can the computer assign feedback on student generated explanations? A comparison study of computer and teacher adaptive guidance. Paper presented at the American Education Research Association Annual Meeting, San Francisco, CA.

Gerard, L.F., Spitulnik, M.W., \& Linn, M.C. (2010). Teacher use of evidence to customize instruction. Journal of Research in Science Teaching, 47(9), 10371063.

Ginns, P. (2006). Integrating information: A meta-analysis of the spatial contiguity and temporal contiguity effects. Learning and Instruction, 16(6), 511-525.

*Gordijn, J., \& Nijhof, W. J. (2002). Effects of complex feedback on computer-assisted modular instruction. Computers \& Education, 39(2), 183-200.

Graesser, A.C., McNamara, D.S., \& VanLehn, K. (2005). Scaffolding deep comprehension strategies through Point \& Query, AutoTutor \& iStart, Educational Psychologist, 40(4), 225-234.

Hattie, J. \& Timperley, H. (2007). The power of feedback. Review of Educational Research. 77(1), 81-112.

Hedges, L. V., \& Olkin, I. (1985). Statistical methods for meta-analysis. New York: Academic Press.

*Heffernan, N. T., Koedinger, K. R., \& Razzaq, L. (2008). Expanding the model tracing architecture: a 3rd generation intelligent tutor for algebra symbolization. International Journal of Artificial Intelligence in Education, 18(2), 153-178.

Herrenkohl, L.R., Tasker, T., \& White, B.Y. (2011) Developing classroom cultures of inquiry and reflection using Web of Inquiry. Cognition and Instruction, 29(1), 144.

Hoffler, T. N., \& Leutner, D. (2007). Instructional animation versus static pictures: A meta-analysis. Learning and Instruction, 17(6), 722-738.

*Huang, T.-H., Liu, Y.-C., \& Shiu, C.-Y. (2008). Construction of an online learning system for decimal numbers through the use of cognitive conflict strategy. Computers \& Education, 50(1), 61-76.

*Hung, P.-H., Hwang, G.-J., Lin, Y.-F., Wu, T.-H., \& Su, I.-H. (2013). Seamless connection between learning and assessment-Applying progressive learning tasks in mobile Ecology inquiry. Educational Technology \& Society, 16(1), 194-205.

Hunter, J. E., \& Schmidt, F. L. (2004). Methods of meta-analysis: Correcting error and bias in research findings (2nd ed.). Thousand Oaks, CA: Sage Publications.

*Kalyuga, S., \& Sweller, J. (2005). Rapid dynamic assessment of expertise to improve the efficiency of adaptive e-learning. Educational Technology Research and 
Development, 53(3), 83-93.

*Kinnebrew, J. S., Biswas, G., \& Sulcer, W. B. (2010). Modeling and measuring selfregulated learning in teachable agent environments. Paper presented at the Association for the Advancement of Artificial Intelligence, Atlanta, GA.

Kluger \& deNisi (1996). The effects of feedback interventions on performance: A historical review, a meta-analysis and a preliminary feedback interventional theory. Psychological Bulletin, 119(2), 254-284.

Koedinger, K. R., \& Aleven, V. (2007). Exploring the assistance dilemma in experiments with Cognitive Tutors. Educational Psychology Review, 19(3), 239-264.

*Koedinger, K. R., McLaughlin, E. A., \& Heffernan, N.T. (2010). A quasi-experimental evaluation of an on-line formative assessment and tutoring system. Journal of Educational Computing Research, 43(4), 489-510.

*Leelawong, K., \& Biswas, G. (2008). Designing learning by teaching agents: The Betty's Brain system. International Journal of Artificial Intelligence in Education, 18(3), 181-208.

Leinhardt, G., \& Steele, M. D. (2005). Seeing the complexity of standing to the side instructional dialogues. Cognition and Instruction, 23(1), 87-163.

Lipsey, M. W., \& Wilson, D. B. (2001). The way in which intervention studies have "personality" and why it's important to meta-analysis. Evaluation and the Health Professions, 24(3), 236-254.

Liu, O. L., Lee, H-S., \& Linn, M.C. (2011) An investigation of explanation multiplechoice items in science assessment, Educational Assessment, 16(3), 164-184.

Mathan, S. A. \& Koedinger, K. (2005). Fostering the intelligent novice: Learning from errors with metacognitive tutoring. Educational Psychologist, 40(4), 257-265.

*McElhaney, K. W., Gerard, L. F., Rafferty, A. N., \& Linn, M. C. (2012). Comparing the Benefits of Automated and Teacher Feedback on Student-generated Molecular Representations. Paper presented at the American Education Research Association, San Francisco, CA.

*McLaren, B. M., DeLeeuw, K. E., \& Mayer, R. E. (2011). Polite web-based intelligent tutors: Can they improve learning in classrooms? Computers \& Education, 56(3), 574-584.

*Mendicino, M., Razzaq, L., \& Heffernan, N. T. (2009). A comparison of traditional homework to computer-supported homework). Journal of Research on Technology in Education, 41(3), 331-359.

*Meyer, B. J., Wijekumar, K., Middlemiss, W., Higley, K., Lei, P. W., Meier, C., \& Spielvogel, J. (2010). Web-based tutoring of the structure strategy with or without elaborated feedback or choice for fifth and seventh grade readers. Reading Research Quarterly, 45(1), 62-92.

*Meyer, B. J. F., Wijekumar, K. K., \& Lin, Y.-C. (2011). Individualizing a web-based structure strategy intervention for fifth graders' comprehension of nonfiction. Journal of Educational Psychology, 1103(1), 140-168.

Minner, D. D., Levy, A. J., \& Century, J. (2010). Inquiry-based science instructionWhat is it and does it matter? Results from a research synthesis years 1984-2002. Journal of Research in Science Teaching, 47(4), 474-496.

*Molenaar, I., Roda, C., van Boxtel, C., \& Sleegers, P. (2012). Dynamic scaffolding of 
socially regulated learning in a computer-based learning environment. Computers \& Education, 59(2), 515-523.

*Narciss, S., \& Huth, K. (2006). Fostering achievement and motivation with bug-related tutoring feedback in a computer-based training for written subtraction. Learning and Instruction, 16(4), 310-322.

NSF (National Science Foundation). (2008). Science and engineering 2008 indicators chapter 1. Mathematics and science teacher quality. http://www.nsf.gov/statistics/seind08/c1/c1s3.htm.

*Poulsen, R., Hastings, P., \& Allbritton, D. (2007). Tutoring bilingual students with an automated reading tutor that listens. Journal of Educational Computing Research, 36(2), 191-221.

*Rafferty, A. N., Gerard, L., McElhaney, K., \& Linn, M. C. (2014). Promoting student learning through automated formative guidance on chemistry drawings. Proceedings of the International Conference of the Learning Sciences (ICLS) 2014 (pp. 386-393).

*Razzaq, L., \& Heffernan, N. T. (2006). Scaffolding vs. hints in the Assistment System. In M. Ikeda, K. Ashley \& T.-W. Chan (Eds.), Proceedings of the 8th International Conference on Intelligent Tutoring Systems (pp. 635-644). Berlin: SpringerVerlag.

*Razzaq, L., \& N. Heffernan. (2009). To tutor or not to tutor: That is the question. In V. Dimitrova, R. Mizoguchi, B. d. Boulay \& A. C. Graesser (Eds.), Artificial Intelligence in Education: Building Learning Systems that Care: From Knowledge Representation to Affective Modeling, Proceedings of the 14th International Conference on Artificial Intelligence in Education, AIED 2009 (pp. 457-464): IOS Press.

*Rebolledo-Mendez, G., R. , Luckin, R., \& du Boulay, B. (2011). Designing adaptive motivational scaffolding for a tutoring system In R. A. Calvo \& S. K. D'Mello (Eds.), New Perspectives on Affect and Learning Technologies (Vol. 3, pp. 155168). New York: Springer.

*Rich, C. S. (2012). The impact of online automated writing evaluation: A case study from Dalian. Chinese Journal of Applied Linguistics, 35(1), 63-79.

Roediger, H. L. \& Karpicke, J. (2005). Test-Enhanced Learning. Psychological Science 17(3), 249-255.

Ruiz-Primo, M. A., \& Furtak, E. M. (2007) Exploring teachers' informal formative assessment practices and students' understanding in the context of scientific inquiry. Journal of Research in Science Teaching, 44 (1), 57-84

*Ryoo, K. \& Linn, M.C. (2014). Comparison of specific and knowledge integration automated guidance for concept diagrams in inquiry instruction. Proceedings of the International Conference of the Learning Sciences (ICLS) 2014, (pp. 585587).

Sao Pedro, M. A., Baker, R., Gobert, J. D., Montalvto, O., \& Nakama, A. (2013). Leveraging machine-learned detectors of systematic inquiry behavior to estimate and predict transfer

*Schoppek, W., \& Tulis, M. (2010). Enhancing arithmetic and word-problem solving skills efficiently by individualized computer-assisted practice. The Journal of Educational Research, 103(4), 239-252. 
*Segedy, J. R., Kinnebrew, J. S., \& Biswas, G. (2012). The effect of contextualized conversational feedback in a complex open-ended learning environment. Education Technology Research and Development, 61(1), 71-89.

Shepard, L.A., (2008). Commentary on the National Mathematics Advisory Panel recommendations on assessment. Educational Researcher, 37(9), 602-609.

Shute, V. J. (2008). Focus on formative feedback. Review of Educational Research, 78(1), 153-189.

*Shute, V. J., Hansen, E. G., \& Almond, R. G. (2008). You can't fatten a hog by weighing it - or can you? Evaluating an assessment for learning system called ACED. International Journal of Artificial Intelligence in Education, 18(4), 289316.

Smith, S. G., \& Sherwood, B. A. (1976). Educational uses of the PLATO computer system. Science, 192, 334-352.

*Tang, J., Rich, C. S., \& Wang, Y. (2012). Technology-enhanced English language writing assessment in the classroom. Chinese Journal of Applied Linguistics, 35(4), 385-399.

*Timms, M. J. (2007). Using Item Response Theory (IRT) to select hints in an ITS. Frontiers in Artificial Intelligence and Applications, 158, 213-221.

VanLehn, K. (2011). The relative effectiveness of human tutoring, intelligent tutoring systems, and other tutoring systems. Educational Psychologist, 46(4), 197-221.

vanZee, E., \& Minstrell, J., (1997). Using questioning to guide student thinking. Journal of the Learning Sciences, 6(2), 227-269.

*Wade-Stein, D., \& Kintsch, E. (2004). Summary street: Interactive computer support for writing. Cognition and Instruction, 22(3), 333-362.

*Walker, E., Rummel, N., \& Koedinger, K. R. (2009). CTRL: A research framework for providing adaptive collaborative learning support. User-modeling and useradapted onteraction. . The Journal of Personalization Research (UMUAI), 19(5), 387-431.

*Walker, E., Rummel, N., \& Koedinger, K. R. (2011). Using automated dialog analysis to assess peer tutoring and trigger effective support. In S. B. G. Biswas \& A. M. J. Kay (Eds.), Proceedings of the 10th International Conference on Artificial Intelligence in Education (pp. 385-393). Berlin: Springer.

*Walker, E., Rummel, N., \& Koedinger, K. R. (2011). Designing automated adaptive support to improve student helping behaviors in a peer tutoring activity. International Journal of Computer-Supported Collaborative Learning, 6(2), 279306.

*White, L., Hixson, N., D’Brot, J., Perdue, J., Foster, S., \& Rhudy, V. (2010). Research brief: Impact of Writing Roadmap 2.0 on WESTEST 2 online writing assessment scores. Charleston, West Virginia: West Virginia Department of Education.

Wilson, M. (2009). Measuring progressions: Assessment structures underlying a learning progression. Journal of Research in Science Teaching, 46(6), 716-730.

*Yun, S., Miller, P. C., Baek, Y., Jung, J., \& Ko, M. (2008). Improving recall and transfer skills through vocabulary building in web-based second language learning: An examination by item and feedback type. Journal of Educational Technology \& Society, 11(4), 158-172. 


\title{
Automated, Adaptive Guidance for K-12 Education
}

\author{
Libby Gerarda, Camillia Matuk ${ }^{b}$, Kevin McElhaneyc \& Marcia C. Linn ${ }^{d}$
}

Re-Submission Date: April 13, 2015

a Libby Gerard [corresponding author]

University of California Berkeley, Graduate School of Education, 4433 Tolman Hall, Berkeley, CA 94720, USA

1-415-794-0525

libby.gerard@gmail.com

b Camillia Matuk

New York University Steinhardt School of Culture, Education, and Human

Development, Media and Games Network, 2 Metrotech Center, Brooklyn, NY 11201, USA

1-646-997-0733

cmatuk@nyu.edu

${ }^{c}$ Kevin McElhaney

SRI International, Center for Technology in Learning, 333 Ravenswood Avenue, Menlo Park, CA 94025, USA

1-510-684-4936

kevin.mcelhaney@sri.com

d Marcia C. Linn

University of California Berkeley, Graduate School of Education, 4611 Tolman Hall, Berkeley, CA 94720, USA

1-510-643-6379

mclinn@berkeley.edu 